\title{
Protocolo para la evaluación del riesgo de colapso de los ecosistemas: Caso de estudio del bosque espinoso (espinal) en la zona central de Chile
}

\author{
Ecosystem collapse risk evaluation protocol: The Espinal \\ (Acacia forest) in the central zone of Chile as case of study
}

\author{
Pliscoff P.,2, Simonetti, J. ${ }^{3}$ y Asmüssen, M. ${ }^{4}$
}

\begin{abstract}
RESUMEN
La biodiversidad enfrenta una crisis a escala global producto de la perdida y fragmentación de los hábitats naturales, lo que está modificando el foco tradicional de las evaluaciones de riesgo desde especies hacia ecosistemas. La Unión Internacional de Conservación de la Naturaleza (IUCN) ha establecido un estándar oficial para la evaluación del riesgo de colapso de los ecosistemas. Proponiendo un modelo conceptual que utiliza cinco criterios basados en las características de distribución y/o función de los ecosistemas. Para ejemplificar la aplicación de esta metodología se seleccionó el ecosistema de "bosque espinoso" de la zona central de Chile, caracterizándolo según: distribución geográfica, biota nativa característica, aspectos abióticos, principales amenazas y procesos claves. La evaluación del riego de colapso pudo ser aplicada a cuatro de los cinco criterios propuestos en el modelo conceptual, lo que permitió establecer que el ecosistema de bosque espinoso sea clasificado bajo la categoría de amenaza de "Vulnerable".
\end{abstract}

Palabras claves: Evaluación de riesgo de Ecosistemas, Espinal, Chile central, colapso.

\begin{abstract}
Biodiversity is facing a crisis at global scale due to fragmentation and loss of natural habitats which are modifying their traditionally evaluation risk focus from species to ecosystems. The International Union for Conservation of Nature (IUCN) established an official standard for assessing the risk of collapse of ecosystems. Proposing a conceptual model that uses five criteria based on the characteristics of distribution and / or function of ecosystems. To exemplify the application of this methodology, the "Thorny forest" ecosystem of the central zone of Chile was selected, characterizing it according to: geographic distribution, characteristic native biota, abiotic aspects, main threats and key processes. The evaluation of the risk of collapse could be applied to four of the five criteria proposed in the conceptual model, which allowed establishing that the Thorny forest ecosystem be classified under the category of "Vulnerable".
\end{abstract}

Keywords: Ecosystem risk assessment, Espinal, central Chile, collapse.

Instituto de Geografía, Pontificia Universidad Católica de Chile, e-mail: pliscoff@uc.cl. Departamento de Ecología, Pontificia Universidad Católica de Chile, e-mail: pliscoff@uc.cl.

Departamento de Ciencias Ecológicas, Facultad de Ciencias Universidad de Chile. e-mail: jsimonett@uchile.cl.

Centro de Ecología, Instituto Venezolano de Investigaciones Científicas, e-mail: marianneasmussen@gmail.com. 


\section{Introducción}

La biodiversidad enfrenta una continua y creciente disminución a nivel global (Tilman et al. 2017). Esta situación pone en peligro tanto la continuidad de las especies como el equilibrio de los ecosistemas, afectando negativamente la sustentabilidad de las poblaciones humanas (Hoekstra et al. 2005; Butchart et al. 2010). La acelerada pérdida de biodiversidad exige nuevas aproximaciones que permitan evaluar e identificar especies y ecosistemas en riesgo de desaparecer (Wilson et al. 2009; Funk \& Fa 2010). Este primer paso es clave para establecer un proceso adecuado de manejo y planificación del territorio, que tenga como objetivo asegurar la conservación de los ecosistemas (Ayensu et al. 1999; Rodriguez et al. 2011; Keith et al. 2013; Keith et al. 2015; Rodriguez et al. 2015; Bland et al. 2016). Los "Libros y Listas Rojas" se han transformado en una efectiva herramienta para sintetizar el estado de conservación de las especies, refinándose conceptualmente desde su creación por la Unión Internacional para la Conservación de la Naturaleza (UICN) hace más de cuarenta años (UICN 2001).

A nivel global, la Lista Roja de UICN ha evaluado 76.000 especies y, a nivel nacional, 109 países han producido Listas Rojas para al menos un taxón (Zamin et al. 2010). Además, las Listas Rojas nacionales son importantes en el ámbito internacional, influenciando tratados y políticas como la Convención sobre el Comercio Internacional de Especies Amenazadas de Fauna y Flora Silvestre (CITES, por sus siglas en inglés), la Convención sobre Especies Migratorias (CMS, por sus siglas en inglés) y el Convenio sobre la Diversidad Biológica (CDB), entre otros (Collen et al. 2013).

Sin embargo, el enfoque de los Libros y Listas Rojas a nivel de especies no es suficiente para evaluar el estado actual de la biodiversidad. Este sistema presenta varias limitaciones como: (1) la evaluación del estado la biodiversidad se restringe solo a uno de sus componentes (Nijman 2005), (2) la tasa de evaluación de especie por especie es relativamente lenta y poco eficiente (Vié et al. 2009), (3) el público tiende a percibir la pérdida de biodiversidad a través de la reducción de servicios provistos por los ecosistemas (agua limpia, alimentos, combustible), y (4) el enfoque en especies no se traduce necesariamente en estrategias de conservación a nivel de paisaje (Ferrier 2002; Cowling et al. 2004). En respuesta a estas limitaciones, el Congreso Mundial de la Conservación resolvió desarrollar los criterios y categorías para una Lista Roja de Ecosistemas (LRE), que buscan complementar las evaluaciones a nivel de especies (Rodriguez et al. 2011).

Para la generación e implementación de una LRE fue necesario resolver importantes retos científicos (Mace et al. 2008). Lo primero fue delimitar el concepto de "ecosistema", el cual posee una gran variedad de definiciones e interpretaciones en la literatura (Tansley 1935; Willis 1997; Olson \& Dinerstein 2002). En este sentido se identificaron cuatro elementos esenciales que están implícitos en el concepto original de Tansley (Pickett \& Cadenasso 2002): 1) un factor biótico o ensamble de especies, 2) un ambiente abiótico asociado, 3) interacciones entre los componentes biótico y abiótico y 4) un espacio físico donde se desarrollan (Bland et al. 2016). Otro gran desafío, fue generar una metodología consistente para evaluar cuantitativamente el proceso de riesgo de pérdida de los ecosistemas, pero que a su vez permita la aplicación de conceptos ecológicos a través de distintos tipos de ambientes (Rodriguez et al. 2011; Keith et al. 2013; Rodriguez et al. 2015; Keith et al. 2015; Bland et al. 2016). 
Actualmente se han desarrollado nuevas teorías que sustentan los modelos conceptuales para la evaluación del riesgo de pérdida de los ecosistemas. Por ejemplo, métodos para manejar la incertidumbre (Akçakaya et al. 2000; Burgman 2005a), medidas ecosistémicas para evaluar el cambio ecológico (Stoddard et al. 2008; Tierney et al. 2009; Patrick et al. 2010), bases de datos temporales sobre la distribución y procesos de los ecosistemas (Curran \& Trigg 2006) y, nuevas teorías relacionadas con la dinámica y función de los ecosistemas (Srivastava \& Vellend 2005; Loreau 2010). El concepto de "función" es considerado un sinónimo de "procesos", haciendo que su estimación sea medible y comparable (Jax 2005). Así, cuando nos referimos a funciones del ecosistema, estamos utilizando un término general que abarca las reservas de materiales (del inglés, stocks, como carbón, agua, nutrientes) y los procesos, que involucran flujos de energía y materia entre los niveles tróficos y el ambiente abiótico (Hooper et al. 2005; Jax 2005). En este contexto, Keith et al. (2013) divulgaron los fundamentos científicos que sustentan la generación de una LRE, con una nueva propuesta que plantea un sólido modelo conceptual. En mayo de 2014, el Consejo de UICN acordó utilizar esta propuesta como un estándar oficial para evaluar el riesgo de colapso de los ecosistemas terrestres, acuáticos continentales y marinos, a nivel global.

El protocolo para la evaluación del riesgo de colapso de los ecosistemas busca sintetizar la gran cantidad de información que puede existir acerca del proceso de degradación de los ambientes naturales y utilizarla para evaluar su estado de conservación. Para lograr dicho objetivo, Keith et al. (2013) propusieron un modelo conceptual en el marco de una evaluación de riesgo, entendiéndose riesgo como la probabilidad de observar un resultado adverso en una escala de tiempo específica (Burgman 2005b). En el contexto de la LRE, el resultado adverso es el colapso del ecosistema, siendo colapso un concepto análogo a la extinción de especies y, por lo tanto, representa el resultado final del proceso de degradación ambiental (Bland et al. 2018). La creación del concepto de colapso obedece a la necesidad de considerar el alto nivel de incertidumbre asociado al proceso de degradación de los ecosistemas y la imposibilidad de declarar que la degradación ambiental puede conducir a la desaparición absoluta de todos los componentes de un ecosistema (Hobbs et al. 2006; Gaston \& Fuller 2008; Nicholson et al. 2009; Keith et al. 2013; Keith et al. 2015; Rodriguez et al. 2015; Bland et al. 2016).

Por ejemplo, diferentes ecosistemas responderán de manera distinta a perturbaciones externas y, en el caso de transformarse en sistemas nóveles, tanto los puntos de transición como la trayectoria que sigan dependerán de diferencias en su variabilidad natural, resiliencia y amenazas (Folke et al. 2004). Por lo tanto, en lugar de definir el colapso como un estado único, irreversible e independiente de la naturaleza del ecosistema, se propone que es un estado caracterizado por haber cruzado el umbral de una o más de las variables que definen su identidad, especialmente su biota nativa característica (BNC) (Bland et al. 2016). Se entiende por BNC al sub-conjunto de especies nativas que distingue a un ecosistema de otro (componente taxonómico) o que juegan un rol importante en la función del ecosistema y la persistencia de otras especies (componente funcional). Quedan excluidas de este concepto todas las especies comunes o nómadas que contribuyen poco a los procesos funcionales del sistema y que probablemente son más comunes en otros ambientes (Bland et al. 2016). Este concepto es clave en la evaluación del riesgo, debido a que su disminución o pérdida representa inequívocamente una consecuencia negativa para la conservación (Convention on Biological Diversity 1992). Así, el proceso de evaluación intenta diagnosticar cómo las distintas amenazas, evaluadas a través de indicadores de cambios en la dis- 
tribución y función de los ecosistemas, alteran la permanencia de este sub-conjunto de especies (Keith et al. 2013; Rodriguez et al. 2015; Keith et al. 2015; Bland et al. 2016).

En este articulo se presenta la aplicación a un ecosistema específico del protocolo de evaluación del riesgo de colapso en ecosistemas desarrollado en el marco de la Lista roja de Ecosistemas (LRE) propuesto por la Unión Internacional de Conservación de la Naturaleza. El ecosistema seleccionado es el Bosque Espinoso (Espinal) presenta en Chile central, el cual ha sido identificado como una zona altamente perturbada tanto por la acción agentes naturales, como por la actividad antrópica (Root-Bernstein et al. 2017), además es uno de los ecosistemas menos representados en el Sistema Nacional de Áreas Silvestres protegidas de Chile (Pliscoff \& Fuentes-Castillo 2011; Luebert \& Pliscoff 2017). La aplicación del protocolo de evaluación de la LRE se ha transformado en un estándar a escala global (Keith et al. 2015), desarrollándose evaluaciones alrededor de todo el mundo tanto en ecosistemas terrestres como marinos. En el caso de Chile, se han realizado evaluaciones a nivel nacional (Pliscoff 2015; Luebert \& Pliscoff 2017), por lo que resulta relevante probar la aplicación del protocolo en un ecosistema especifico, para evaluar la aplicación de los criterios propuestos y realizar comparaciones con los análisis realizados a mayor escala. Finalmente, el poseer distintos tipos de evaluaciones, tanto a nivel de escala espacial como de distintas zonas geográficas, permitirá generar más antecedentes para consolidar la aplicación de una Lista roja de ecosistemas en Chile.

\section{Metodología}

\section{Caso de Estudio: El Espinal}

El "bosque espinoso" o "Espinal" es uno de los ecosistemas naturales más amenazados de la zona central de Chile. Aunque se ha planteado que este bosque corresponde a una fase de degradación del bosque esclerófilo (Oberdorfer 1960), actualmente esto resulta improbable considerando las condiciones bioclimáticas en que se desarrolla (Luebert \& Pliscoff 2017). Antecedentes históricos demuestran que este ecosistema ha sufrido fuertes procesos de degradación, principalmente por deforestación y la presencia de especies invasoras. Se plantea que la cobertura original del bosque espinoso alcanzó una superficie de $3.425 \mathrm{~km}^{2}$, de los cuales actualmente sólo persiste el $38,4 \%\left(1.316 \mathrm{~km}^{2}\right)$. La acción del ganado y conejos ha afectado los procesos naturales de depredación de semillas y plántulas, cambiando la estructura original de este tipo de bosque. Por estas razones, consideramos al bosque espinoso un ecosistema natural que se encuentra fuertemente amenazado, siendo un excelente candidato para evaluar el modelo de riesgo de colapso, a través de los criterios y categorías de la Lista Roja de Ecosistemas de UICN (Bland et al. 2016). Como primera etapa se recopilo información ya existente sobre distintas clasificaciones del ecosistema, se describió su biota nativa característica, aspectos del Ambiente abiótico, su distribución espacial, la presencia de Procesos claves (naturales y antrópicos) y finalmente las principales interacciones y amenazas asociadas.

\section{Estructura del protocolo}

Para evaluar el riesgo de colapso de un ecosistema, Keith et al. (2013) y posteriormente Bland et al. (2016) propusieron usar cinco criterios, basados en una o más variables sucedáneas (del inglés, proxy), que están asociadas a características de distribución y función, respondiendo a 
los cuatro factores que se identifican en el concepto de ecosistema antes mencionado (Fig. 1): A) tasas de declive en la distribución del ecosistema, B) distribuciones restringidas que están sometidas a una continua declinación o amenaza, C) tasas de degradación ambiental (elementos abióticos), D) tasas de disrupción de los procesos bióticos y, E) estimaciones cuantitativas del riesgo de colapso - este último integra los múltiples procesos de degradación. Un ecosistema bajo estudio debe ser evaluado utilizando todos los criterios para los cuales existen datos disponibles. El estatus de amenaza final corresponderá al nivel más alto de amenaza indicado por cualquiera de los criterios, ya que el riesgo es determinado por el factor más limitante. Los criterios y umbrales asignan a los ecosistemas una de ocho categorías: dos que representan "no amenaza" (LC y NT), tres de amenaza (CR, EN, VU), una para ecosistemas colapsados (CO), una para falta de datos (DD) y una para ecosistemas no evaluados (NE) (Fig. 2).

Figura 1. Mecanismos a través de los cuales un ecosistema puede alcanzar el estado de colapso, en función de los síntomas del riesgo de colapso.

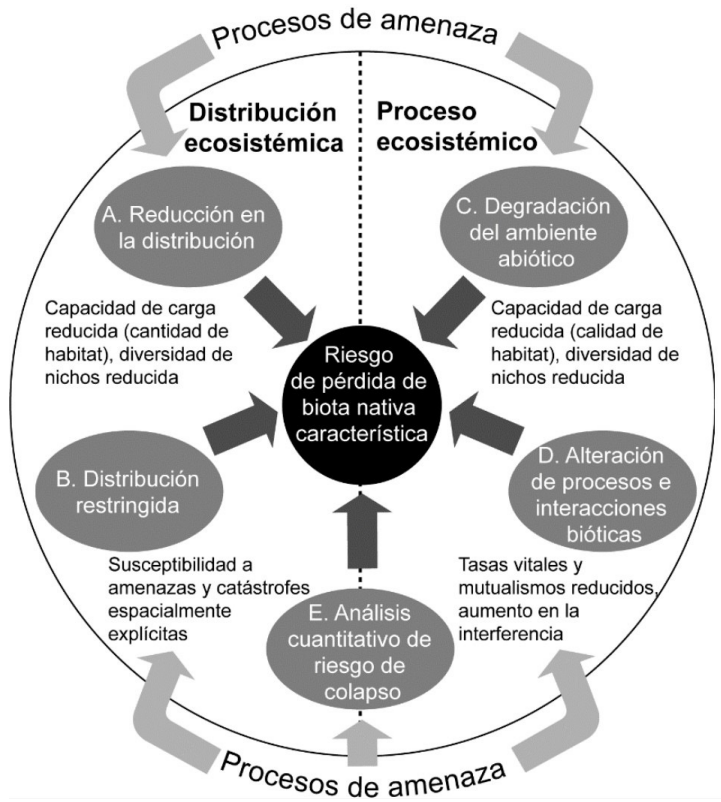

Fuente: (Bland et al. 2016)

Figura 2. Estructura de las categorías para una Lista Roja de Ecosistemas de UICN.

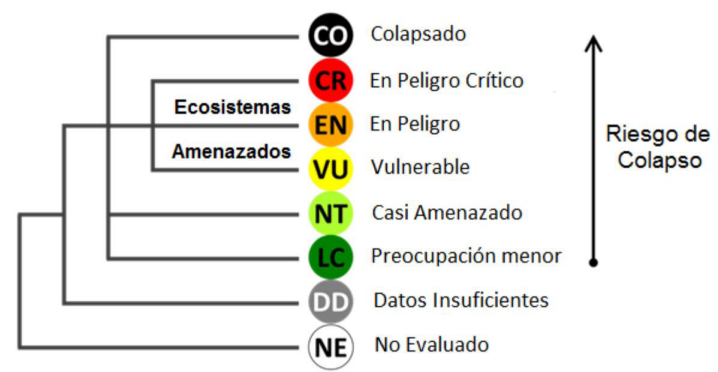

Fuente: (Bland et al. 2016) 


\section{Escalas temporales}

Los criterios evalúan la degradación dentro de tres marcos temporales: actual, futuro e histórico (Fig. 3). Las degradaciones actuales son evaluadas abarcando los últimos 50 años: lo suficientemente recientes para detectar tendencias actuales y lo suficientemente largas como para poder diagnosticar cambios direccionales de manera confiable. La evaluación de la degradación futura requiere de predicciones sobre los cambios que ocurrirán en los próximos 50 años, o para cualquier lapso de 50 años que comprenda el presente y el futuro. La degradación histórica se evalúa en relación al estatus del ecosistema en la fecha de referencia hipotética de 1750, ya que este año se corresponde aproximadamente con el inicio de la explotación de los ecosistemas a una escala industrial (Bland et al. 2016).

Figura 3. Escalas temporales para la evaluación de los criterios A, C y D (Keith et al. 2013)

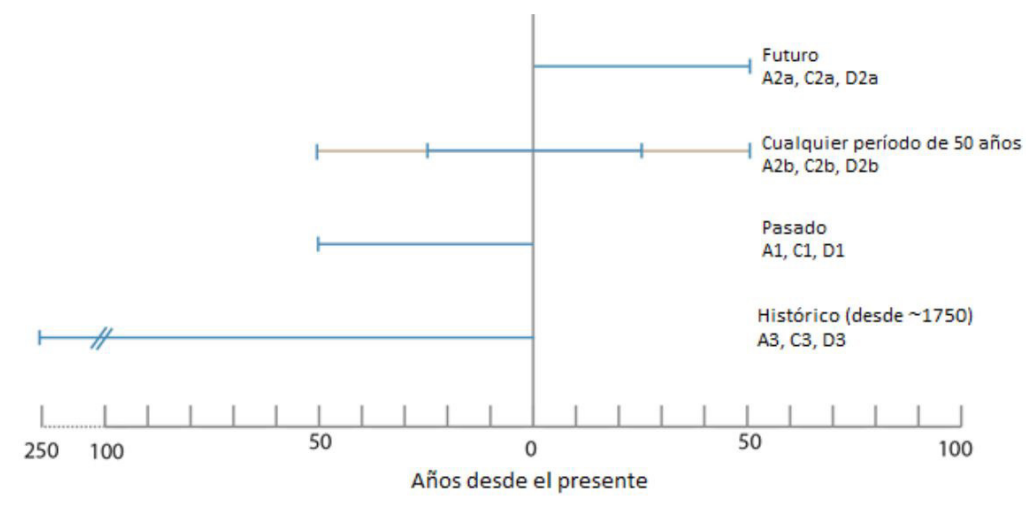

Fuente: (Bland et al. 2016)

\section{Umbrales de degradación y colapso}

Las categorías de riesgo de colapso son definidas por distintos umbrales de degradación. La lógica de dichos umbrales es teórica y pragmática, reconociendo que 1) la teoría provee una base cualitativa para establecer umbrales de degradación ordenados, pero provee una guía limitada al momento de establecer sus valores absolutos; y 2) el objetivo es clasificar los ecosistemas en categorías de riesgo ordinales informativas, más que estimar probabilidades de colapso precisas (Bland et al. 2016). Por consiguiente, para los criterios que involucran la reducción del área o funcionamiento de un ecosistema ( $A, C$ y D), se fijaron valores umbrales a intervalos relativamente uniformes para las degradaciones actuales y futuras (Vulnerable 30\%, En Peligro 50\%, En Peligro Crítico $80 \%$ ). El establecimiento del intervalo de los umbrales entre $0 \%$ y $100 \%$ busca asignar los ecosistemas dentro de las categorías de riesgo de manera informativa y no altamente sesgada, mientras que el umbral más bajo de $30 \%$ reconoce que son necesarias evidencias de una reducción apreciable en la distribución o funcionamiento del ecosistema para justificar su clasificación dentro de una categoría de amenaza. Estos umbrales "basales" son consistentes con los umbrales 
establecidos para la reducción poblacional de los criterios de la Lista Roja de especies (UICN 2001). Para disminuciones históricas, Bland et al. (2016) establecieron umbrales más altos $(50 \%$, $70 \%, 90 \%$ ) ya que las escalas temporales son más largas (Cuadro 1).

Por otra parte, cada uno de los cinco criterios implica un umbral de colapso (Fig. 1). Para los criterios basados en la extensión espacial (A y B), generalmente se puede suponer que un ecosistema ha colapsado si su distribución se reduce a cero. Sin embargo, el uso del umbral 'cero' dependerá de las variables y mapas utilizados para representar la distribución del ecosistema, y algunos ecosistemas pueden colapsar antes de que su distribución mapeada disminuya a cero. Para aquellos criterios basados en variables funcionales ( $C$ y $D$ ), usualmente un intervalo de valores definirá el colapso para una variable determinada. Este intervalo debería estar acotado entre el valor mínimo posible, donde no cabe duda que el sistema ha colapsado, y un máximo factible basado en observaciones de casos locales donde el ecosistema parece haber cambiado más allá de su intervalo de variación (definido en la descripción de su biota nativa característica y sus procesos), y como resultado ha perdido su biota nativa característica. Un enfoque similar puede ser aplicado cuando se utilizan modelos de simulación para estimar el riesgo de colapso bajo el criterio E. En este caso, deben identificarse los estados colapsados entre la variedad de estados representados por el modelo, y deben especificarse los umbrales acotados de abundancia relativa y/o persistencia que permiten identificar los límites de variación natural en el sistema (Cuadro 1). 


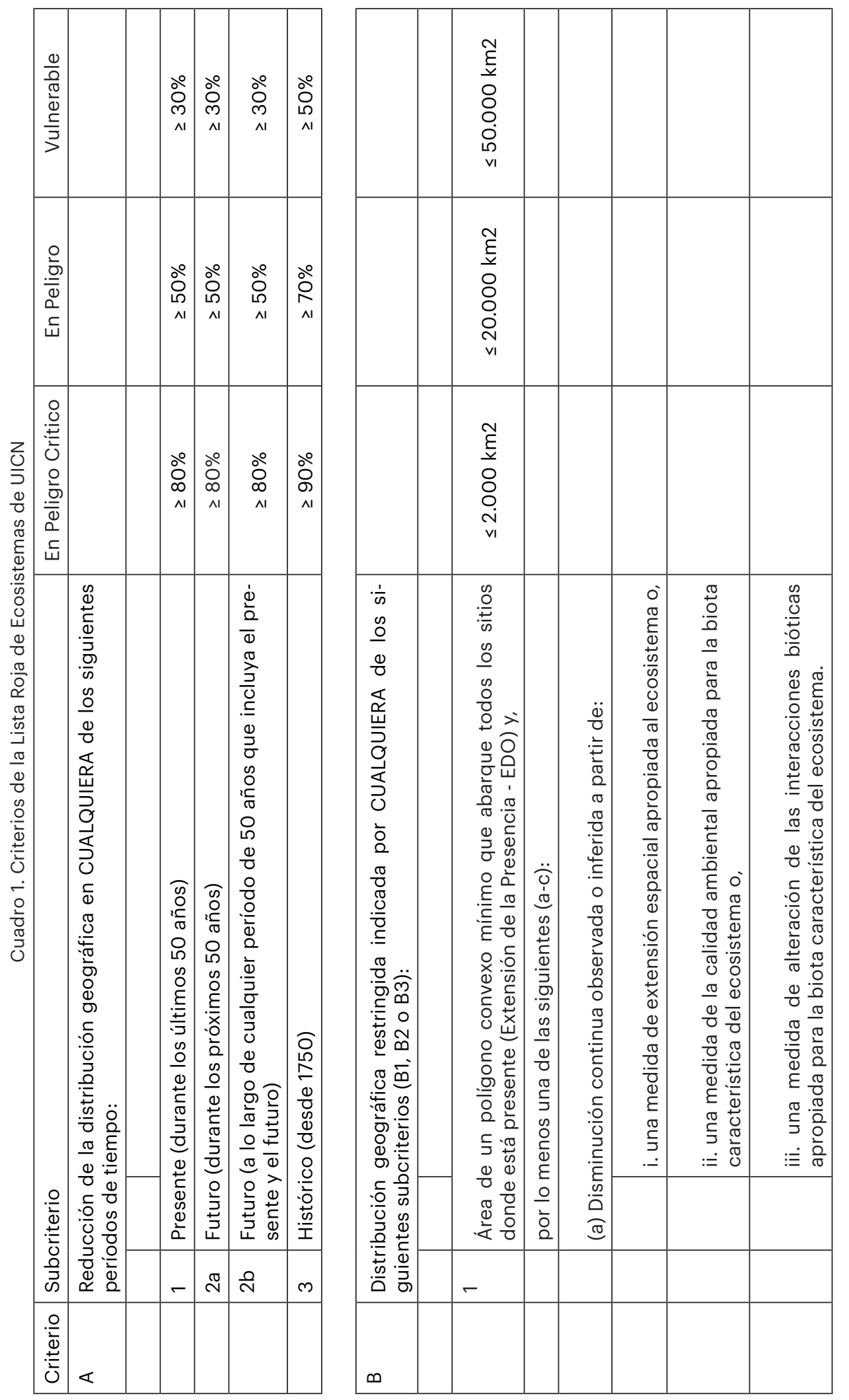




\begin{tabular}{|c|c|c|c|c|c|c|}
\hline $\begin{array}{l}\frac{0}{0} \\
\frac{\pi}{\frac{\pi}{0}} \\
\frac{5}{3} \\
\frac{5}{5}\end{array}$ & & $\begin{array}{l}\mathscr{0} \\
\frac{0}{0} \\
\frac{\pi}{0} \\
\frac{0}{\pi} \\
\frac{0}{0} \\
\frac{0}{0} \\
\frac{0}{v_{1}}\end{array}$ & $\begin{array}{l}\stackrel{\circ}{\circ} \\
\text { vi }\end{array}$ & & & $?$ \\
\hline 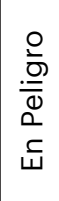 & & 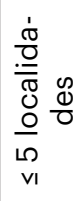 & $\underset{\mathrm{v} 1}{\stackrel{\bigcirc}{2}}$ & & & 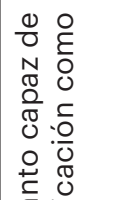 \\
\hline 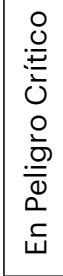 & & 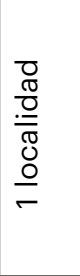 & $\underset{v 1}{N}$ & & & 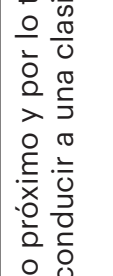 \\
\hline & 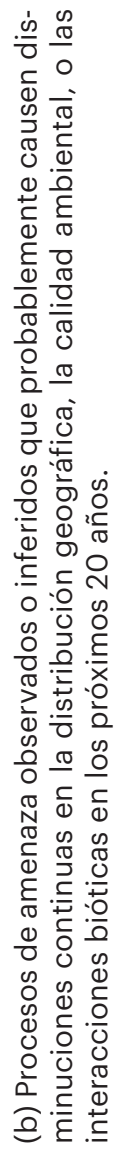 & 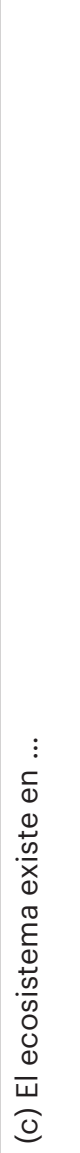 & 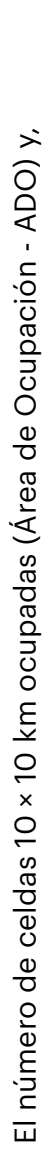 & 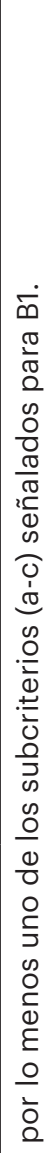 & 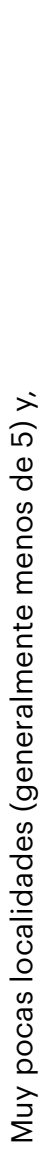 & 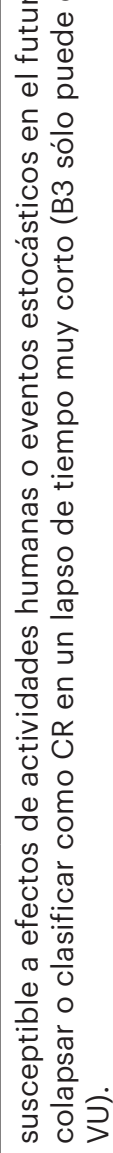 \\
\hline & & & $N$ & & $m$ & \\
\hline & & & & & & \\
\hline
\end{tabular}

\begin{tabular}{|c|c|c|c|c|c|}
\hline 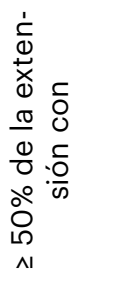 & 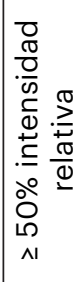 & 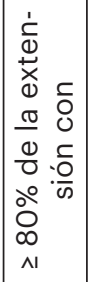 & 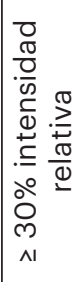 & 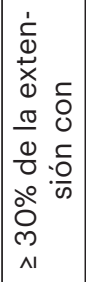 & 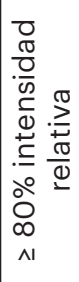 \\
\hline 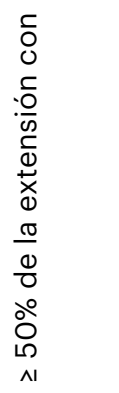 & 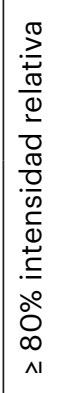 & 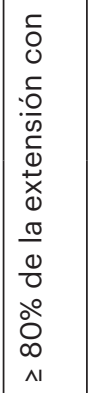 & 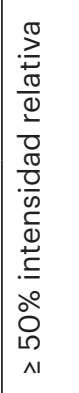 & & \\
\hline 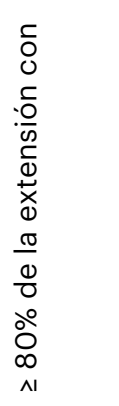 & 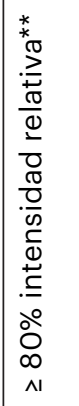 & & & & \\
\hline 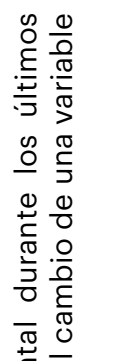 & & & & & \\
\hline 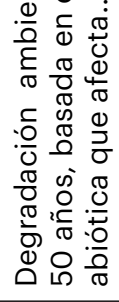 & & & & & \\
\hline- & & & & & \\
\hline 0 & & & & & \\
\hline
\end{tabular}




\begin{tabular}{|c|c|c|c|c|c|c|c|c|c|}
\hline 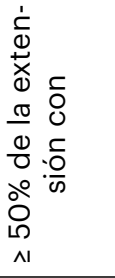 & 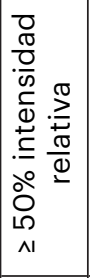 & 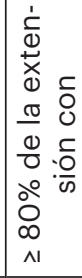 & 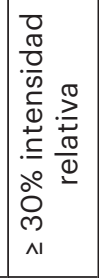 & 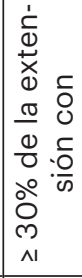 & 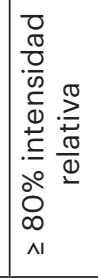 & 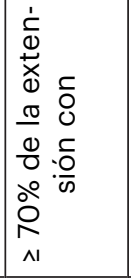 & 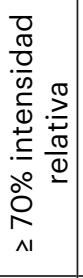 & & 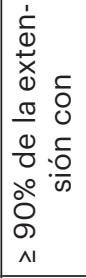 \\
\hline 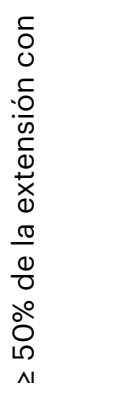 & 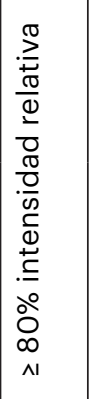 & 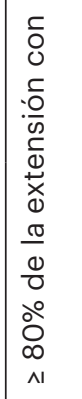 & 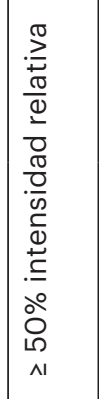 & & & 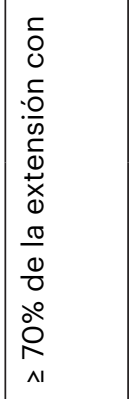 & 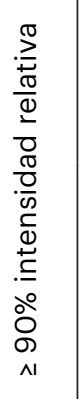 & & 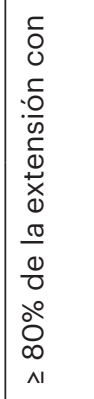 \\
\hline 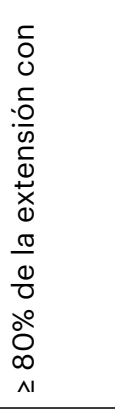 & 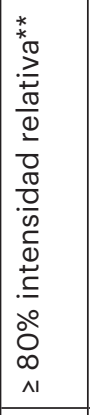 & & & & & 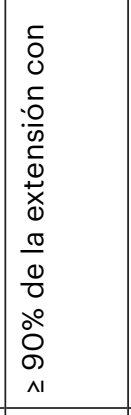 & 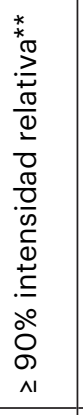 & 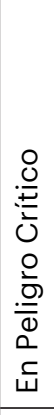 & \\
\hline 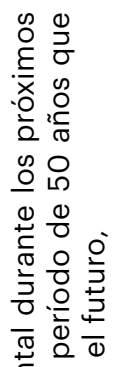 & 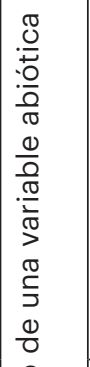 & & & & & 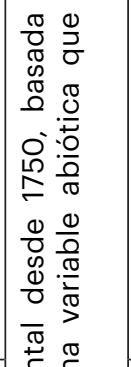 & & & \\
\hline 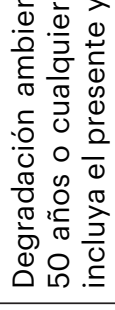 & 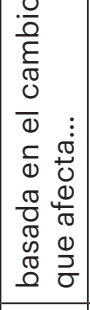 & & & & & 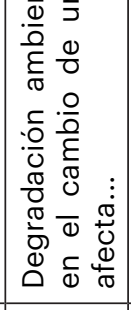 & & & \\
\hline$N$ & & & & & & m & & & \\
\hline
\end{tabular}




\begin{tabular}{|c|c|c|c|c|c|c|c|c|c|c|c|c|}
\hline 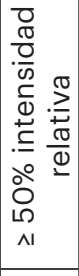 & 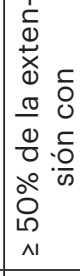 & 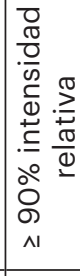 & 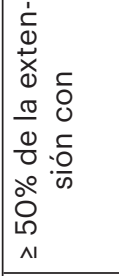 & 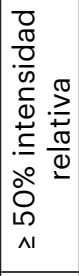 & 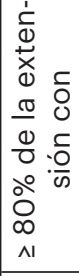 & 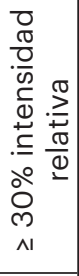 & 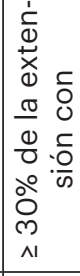 & 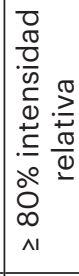 & 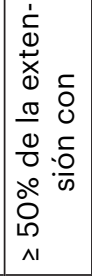 & 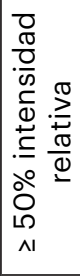 & & 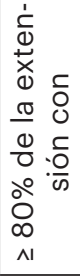 \\
\hline 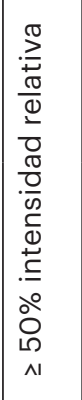 & & & 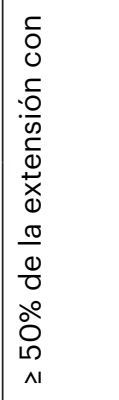 & 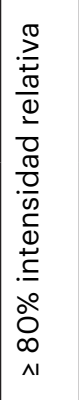 & 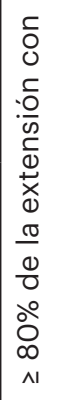 & 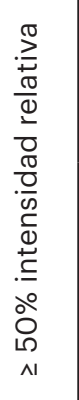 & & & 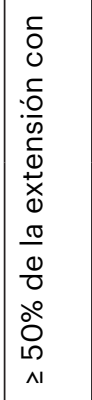 & 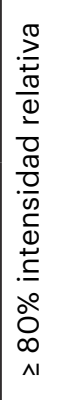 & & 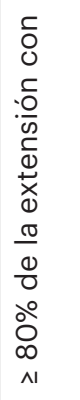 \\
\hline & & & 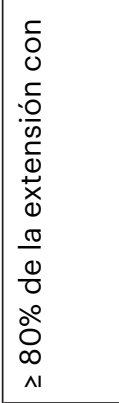 & 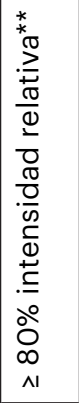 & & & & & 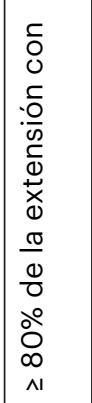 & 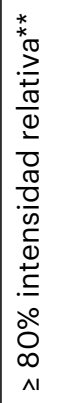 & & \\
\hline & & & 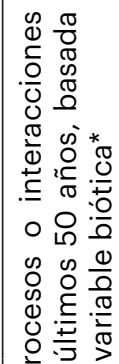 & & & & & & 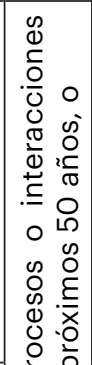 & 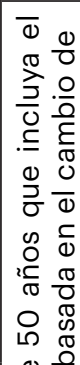 & 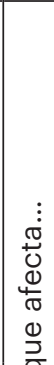 & \\
\hline & & & 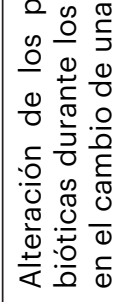 & 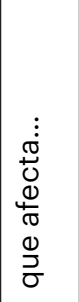 & & & & & 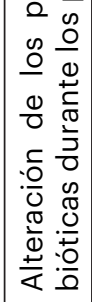 & 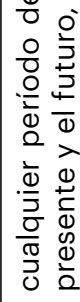 & 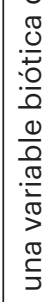 & \\
\hline & & & - & & & & & & $N$ & & & \\
\hline & & & 0 & & & & & & & & & \\
\hline
\end{tabular}




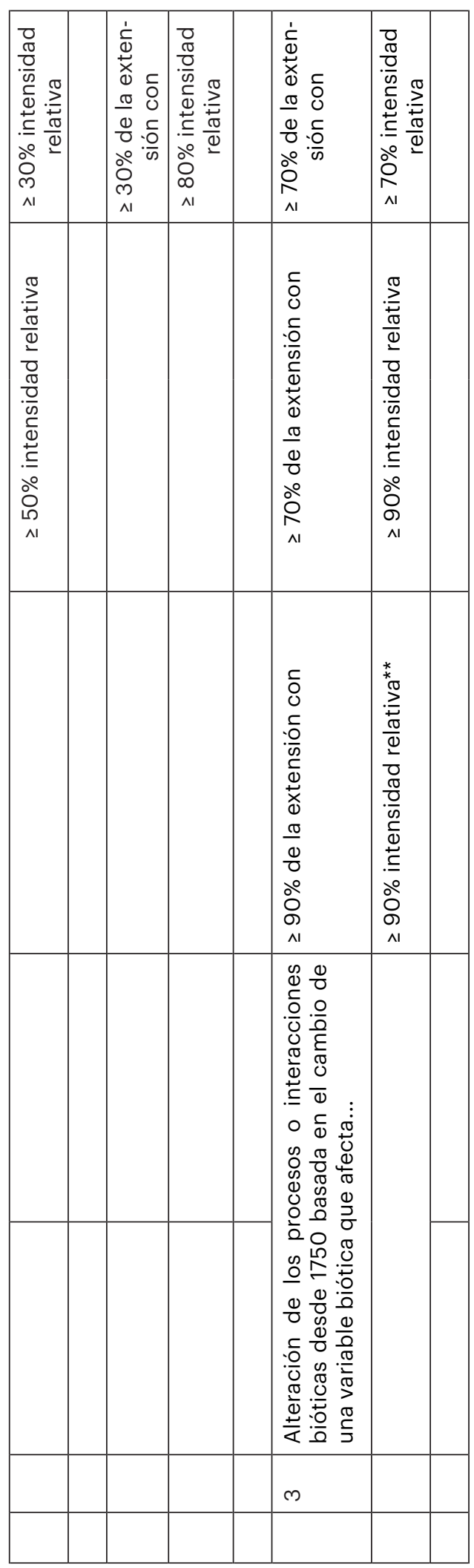

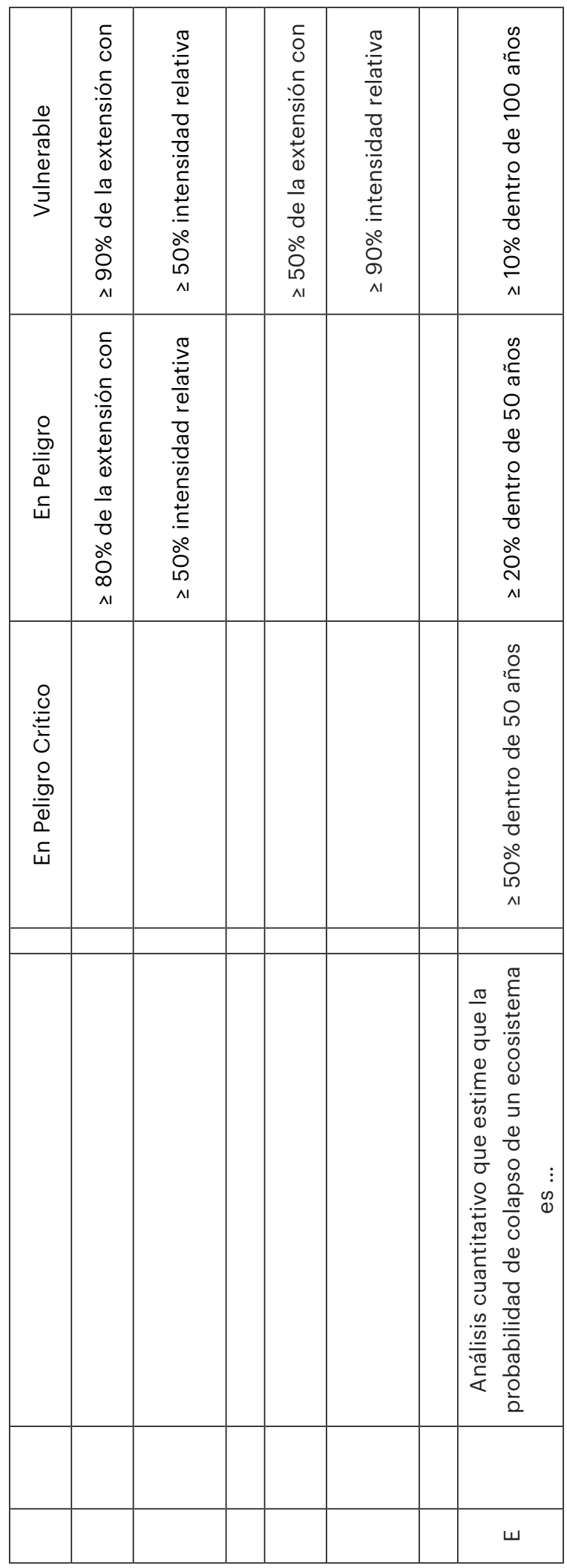




\section{Resultados}

\section{Descripción del ecosistema}

\section{Clasificaciones}

A nivel nacional, el bosque espinoso ha sido descrito como una unidad vegetacional definida por la fisonomía de "sabana" (Ovalle \& Godron 1989), donde se identifica una vegetación dominada por árboles en un dosel abierto. Oberdorfer (1960) incluye al bosque espinoso como una asociación (Cestro-Trevoetum) definida por la presencia de las especies arbustivas acompañantes características en su distribución interior. Gajardo (1994), reconoce al bosque espinoso dentro de la sub-región del "Matorral" y del "Bosque Espinoso", dando cuenta de un carácter mixto en términos fisionómicos. Luebert \& Pliscoff (2017) reconocen el carácter de formación vegetacional de bosque en todas las unidades adscritas al bosque espinoso, diferenciándolo en función de su composición florística y distribución geográfica. Los autores reconocen cuatro unidades dentro de la formación de "Bosque espinoso dominado por Acacia caven": (1) el Bosque espinoso mediterráneo interior de Acacia caven y Prosopis chilensis, (2) Bosque espinoso mediterráneo andino de Acacia caven y Baccharis paniculata, (3) Bosque espinoso mediterráneo costero de Acacia caven y Maytenus boaria y (4) Bosque espinoso mediterráneo interior de Acacia caven y Lithraea caustica.

A nivel internacional, se considera parte de la Ecoregión del Matorral Chileno (Chilean Matorral), la cual se clasifica dentro de los Bosques, Montes y Matorrales Mediterráneos según la clasificación de Ecoregiones Terrestres de WWF (Olson \& Dinerstein 2002) y, según el esquema de clasificación de hábitats de la UICN (versión 3.1), el ecosistema es clasificado como "2. Sabanas/2.1. Sabanas secas" (Dry Savannas).

\section{Biota nativa característica}

El bosque espinoso corresponde a una formación boscosa dominada por la presencia de la especie Acacia caven (espino) (Fig. 4). En términos de su fisionomía, esta formación vegetacional se presenta con una estrata arbórea de individuos aislados sin formar un bosque continuo, aunque su grado de cobertura depende de las condiciones del sitio y mesoclimáticas. Entre las especies arbóreas acompañantes de Acacia caven se pueden identificar Prosopis chilensis, Quillaja saponaria y Lithraea caustica, en su distribución norte, y Kageneckia oblonga y Maytenus boaria, en su distribución sur. La estrata arbustiva presente dentro del bosque espinoso es muy diversa. Está compuesta principalmente por Colliguaja odorifera, Retanilla trinervia, Trevoa quinquenervia, Cestrum parqui, Muehlenbeckia hastulata, Schinus polygamus, Solanum ligustrinum y Proustia cuneifolia. Por otra parte, la estrata herbácea posee la característica de ser muy abundante durante la primavera, donde se puede identificar especies como Avena barbata, Bromus berteroanus, Vulpia myuros, Centaurea melitensis, Helenium aromaticum, Moscharia pinnatifida y Phacelia brachyantha. En cuanto a la presencia de fauna, se han detectado micro-mamíferos como Oligoryzomys longicaudatus, Octodon degus y Abrothrix olivaceus (Muñoz-Pedreros et al. 2010). También especies de aves rapaces (Geranoaetus melanoleucus, Parabuteo unicinctus) han sido reconocidas como un elemento importante dentro de este bosque (Pavez et al. 2010). 
Figura 4. Bosque Espinoso de Chile Central, fotografía de Andrés Moreira.

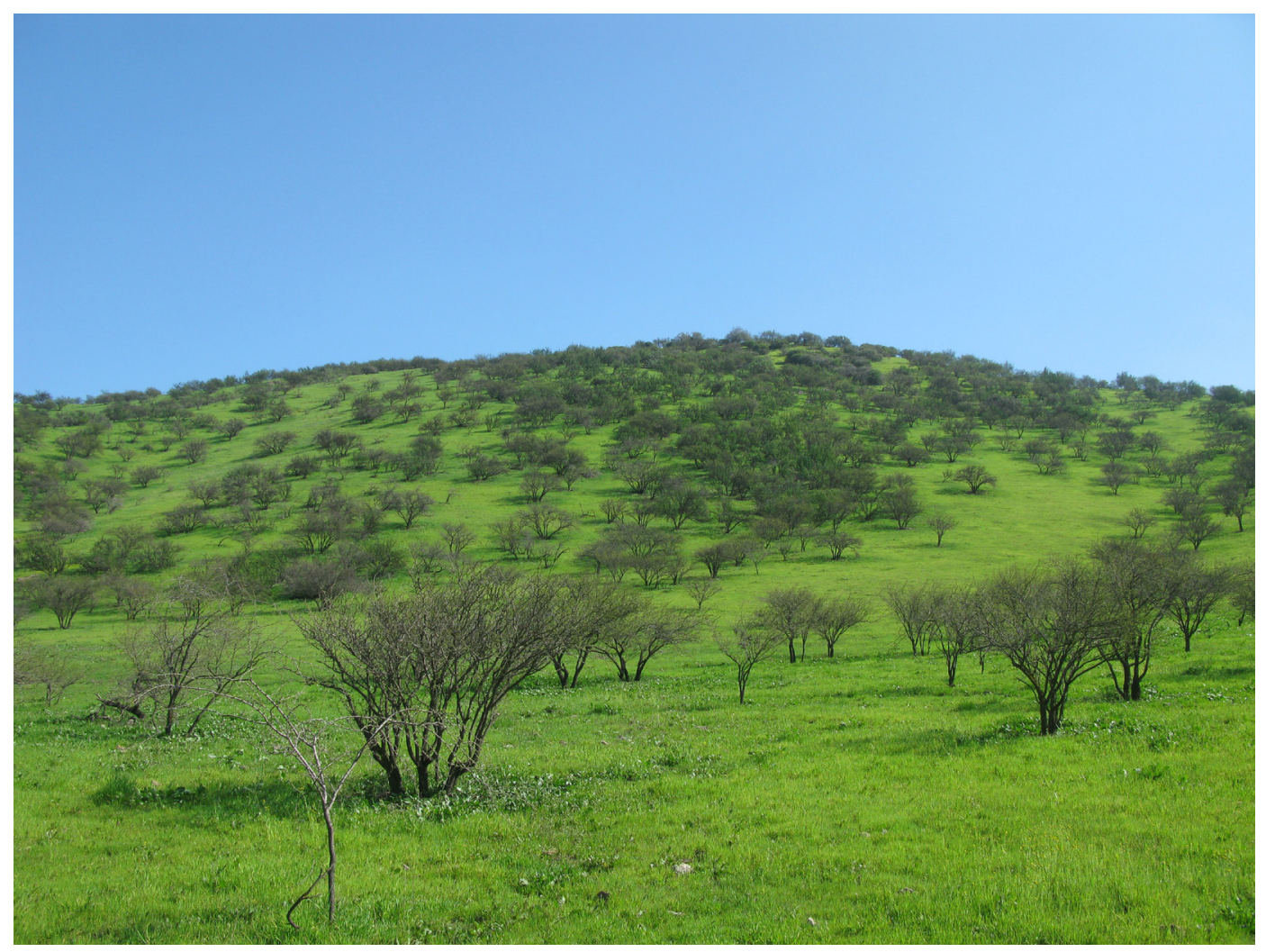

\section{Ambiente abiótico}

El bosque espinoso se presenta dentro del macrobioclima Mediterráneo en su variante pluviestacional-oceánica (Luebert \& Pliscoff 2017). Esto corresponde a zonas caracterizadas por un fuerte contraste estacional en los patrones térmicos y de precipitación. Se pueden identificar principalmente dos épocas, una estival cálida con sequía y una invernal fría, donde se concentran las precipitaciones. En términos de su meteorología, los rangos de temperatura anual promedio oscilan entre los 13 y los $17^{\circ} \mathrm{C}$, identificándose las temperaturas más bajas en el mes de Julio y las más altas en el mes de Enero (Barros et al. 2007). La precipitación anual promedio varía en el gradiente latitudinal entre los 100 y los $1.000 \mathrm{~mm}$, concentrándose las precipitaciones entre los meses de julio y agosto, variando la estación cálida con sequía desde los 10 meses en la zona norte a 3 a 5 en la zona sur de la distribución (Barros et al. 2007). En términos de su distribución espacial relacionada con el relieve y las geoformas, este ecosistema se ubica en la zona de la depresión intermedia, con excepción de la presencia en un área relacionada a las planicies costeras en el extremo sur de la región de Valparaíso y al norte de la región de O'Higgins, principalmente en sectores planos o de pendiente suave de la depresión intermedia (200-800 m). Los suelos en los cuales se desarrolla el ecosistema, varían desde texturas livianas a medias, características 
de suelos profundos, hasta suelos más delgados y pedregosos con bajo contenido de materia orgánica. Finalmente, en la zona costera de su distribución (Región de Valparaíso y O'Higgins), se presenta en suelo granítico de texturas livianas medias (Serra 1997).

\section{Distribución}

Se distribuye entre los $31^{\circ}$ y los $37^{\circ}$ grados de Latitud sur. Está presente en las laderas bajas (pie-de-monte) de la cordillera de Los Andes, en las regiones Metropolitana y del Libertador Bernardo O'Higgins, entre 600 y 1.200 m de altitud. Además, se encuentra en los lomajes costeros del sur de la región de Valparaíso y en el norte de la del Libertador Bernardo O'Higgins, entre 0 y $500 \mathrm{~m}$ de altitud. En su distribución más al sur, se observa en las planicies aluviales de la depresión intermedia de la región del Libertador Bernardo O'Higgins y la del Maule, entre 100 y 900 m de altitud (Fig. 5).

\section{Procesos claves (naturales y antrópicos), interacciones y amenazas}

Aunque sabemos que los ecosistemas mediterráneos de Chile son clasificados dentro de los ambientes más amenazados, tanto a nivel nacional como internacional, existe un gran vacío de información acerca de los procesos claves que determinan la dinámica dentro de dichos ecosistemas (Arroyo et al. 2000; Myers et al. 2000). En particular para el caso del bosque espinoso, la información disponible se enfoca más en los procesos de manejo y en las amenazas que actualmente enfrenta. En este contexto, existe fuerte evidencia que la extracción histórica e intensa intervención para la obtención de material con fines dendroenergéticos, ha generado un impacto altamente negativo en la estructura del bosque, modificando tanto la abundancia como la arquitectura de los individuos. Por ejemplo, Acacia caven en condiciones favorables y sin intervención, puede presentar alturas de $7 \mathrm{~m}$ y diámetros de más de $50 \mathrm{~cm}$ (Cornejo \& Gándara 1980). Sin embargo, producto del continuo aprovechamiento al que ha sido sometida esta especie, lo más común es encontrarla como arbustos jóvenes, retoños e individuos nuevos. Este tipo de arquitectura, se ha demostrado que tiene efectos sobre la composición florística de la estrata herbácea. La participación de gramíneas decae abruptamente como consecuencia de la intervención y aumentan especies leguminosas, compuestas y geraniáceas de ciclos biológicos más cortos, debido a las nuevas condiciones ambientales (hídricas y tróficas) más restrictivas (Squella \& Soto 1993).

Por otra parte, el sobrepastoreo y la consecuente depredación de semillas y plántulas por animales como conejos y ganado, también han contribuido a la degradación y reestructuración del bosque espinoso (Fuentes et al. 1989; Gutiérrez et al. 2007). La presencia de escombros y basura en algunas áreas de su distribución, indican que las especies dominantes podrían enfrentar severas limitaciones en su reclutamiento, debido al daño mecánico que podrían experimentar las plántulas simplemente al verse aplastadas por este tipo de materiales (Valdivia \& Romero 2013). Al mismo tiempo, los depósitos de basura han contaminado las aguas de las napas subterráneas, lo que dificulta aún más el posible reclutamiento y establecimiento de este tipo de árboles, ya que como habita en climas semiáridos, el agua es un recurso limitante (Gutiérrez et al. 2007). 
Figura 5. Distribución potencial y actual del Espinal (Luebert y Pliscoff 2017)
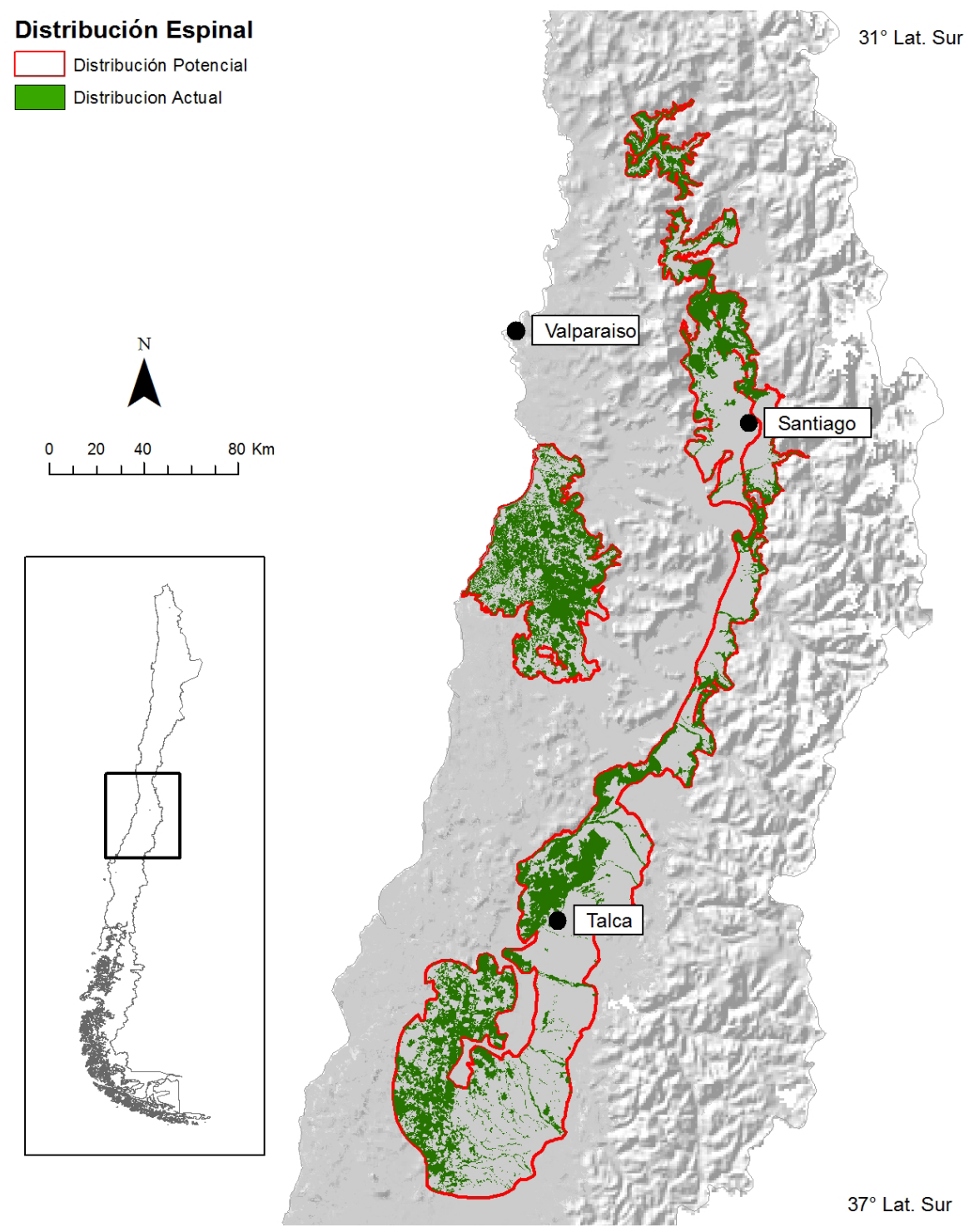

Fuente: Elaboración propia

\section{Modelo conceptual}

El modelo conceptual del bosque espinoso (Fig. 6), presenta los procesos y amenazas claves, identificando sus interrelaciones, los cuales derivan en dos indicadores (aumento de erosión y desertificación y, cambio en la estructura y composición de especies de flora y fauna) que definirían una condición probable de colapso del ecosistema. 
Figura 6. Modelo conceptual desarrollado para el bosque espinoso.

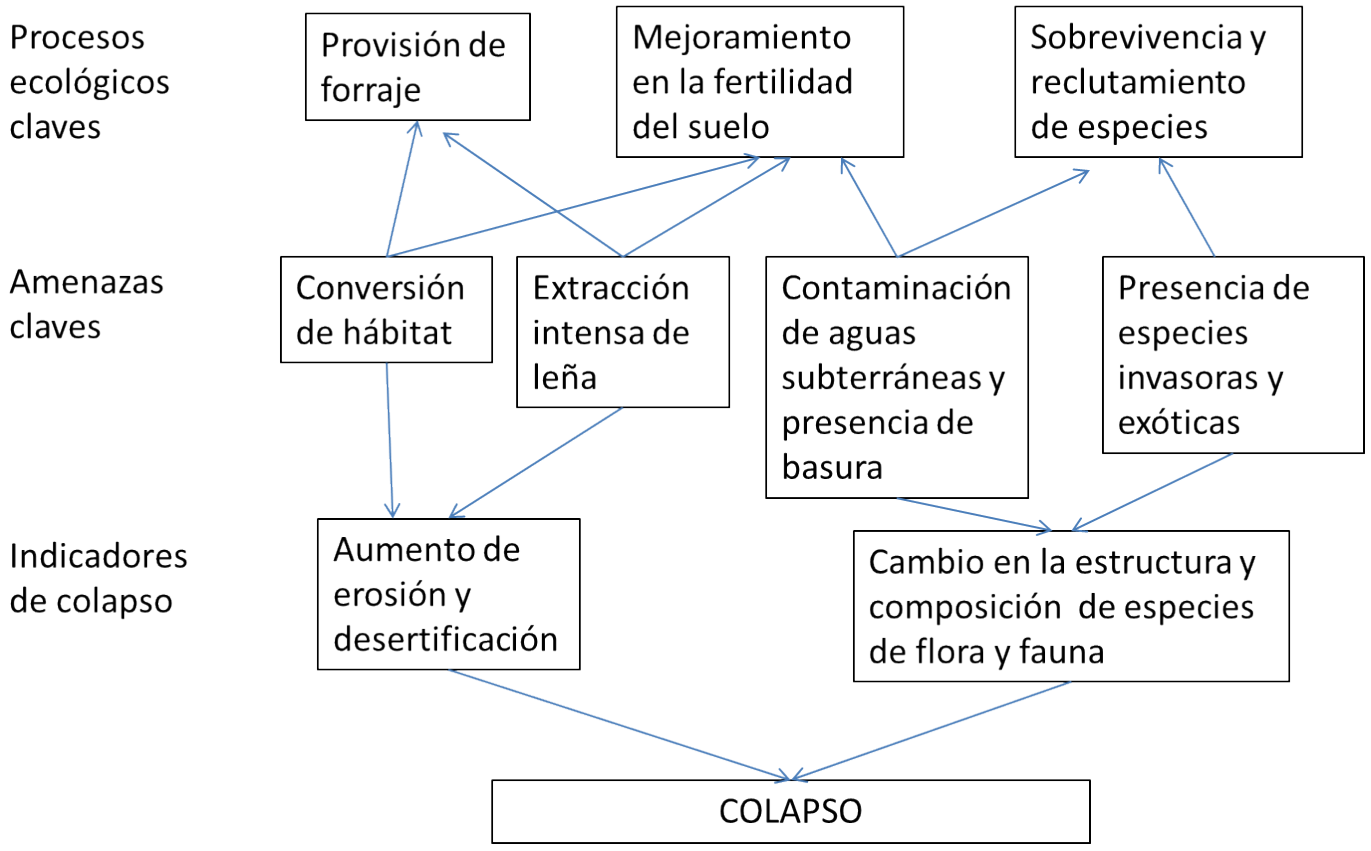

Fuente: Elaboración propia

\section{Aplicación de criterios de evaluación}

\section{Criterio A - Declinación en la distribución:}

A medida que el área de un ecosistema se reduce, la pérdida de biota resultante depende de su patrón espacial en relación con las amenazas y las medidas de conservación. Este análisis se planteó en dos periodos de tiempo que definen los subcriterios; el primero implica la reducción en la distribución, a lo largo de cualquier periodo de 50 años que incluya el presente y el futuro (A2b), y el último, es la disminución histórica (A3) que se calcula desde el año 1750. No fue posible incorporar el primer subcriterio A1, debido a la imposibilidad de contar con información histórica reciente (últimos 50 años) del cambio de uso de suelo en el área del bosque espinoso.

En el caso del subcriterio A2b, se realizó una estimación basada en una proyección de la tasa de pérdida reciente, calculada en los últimos 20 años (1992-2012). Se calculó la superficie remanente con vegetación natural para el año 1992 y para el año 2012, combinando información de cobertura de uso de suelo, a partir del Catastro forestal de INFOR y de coberturas de uso de suelo agrícola y urbano, obtenidas de imágenes satelitales MODIS (Pliscoff 2015). A partir de la diferencia de superficie remanente de los años 1992 y 2012, se calculó la tasa de pérdida en los 
últimos 20 años, la cual fue extrapolada en forma lineal para los próximos 30 años, usando la tasa de pérdida calculada para cada ecosistema en el período 1992-2012. Se aplicó la tasa de pérdida de esta forma porque no se cuenta con antecedentes que permitan estimar distintas tasas de pérdida en zonas geográficas particulares del país.

El subcriterio A3, por su parte, se calculó con la diferencia de superficie entre la distribución potencial del piso de vegetación, definida por Luebert \& Pliscoff 2017 (asumiendo que correspondería a una fecha aproximada al año 1750) y la superficie remanente actual de cada piso de vegetación (2014), esta última calculada con los mismos elementos que en el subcriterio A2a.

\section{Criterio B - Tamaño de distribución restringida}

Este criterio busca identificar aquellos ecosistemas cuya distribución se encuentra tan restringida que están en riesgo de colapso por la concurrencia de eventos o procesos amenazantes. Este criterio se subdivide en tres subcriterios. El primer subcriterio B1, utiliza una medida de "Extensión de la Ocurrencia (EDO)" que se define a partir de un polígono convexo mínimo que comprende todas las instancias del ecosistema, definiéndose a partir de la disminución continúa observada o inferida (B1a); procesos de amenaza observados o inferidos (B1b), y si el ecosistema existe en más de una localidad (B1C). El segundo subcriterio B2, abarca el concepto de "Área de Ocupación (ADO)", el cual calcula el número de celdas ocupadas en una cuadrícula. El criterio $B$ se aplicó en uno de los subcriterios presentados: el criterio B2, referido al número de celdas 10 $\times 10 \mathrm{~km}$ ocupadas (ADO). El tercer subcriterio B3, se define en función del número de presencias puntuales del ecosistema evaluado.

Para el caso del bosque espinoso, el subcriterio B1 se aplicó calculando un polígono mínimo convexo (Fig. 6) y se estimó la categoría en función del subcriterio condicionante de disminución continua (a) por una medida de extensión espacial (i). En este caso, se revisó el comportamiento en el criterio de reducción espacial A2b. B2 fue aplicado utilizando la distribución actual de cada piso de vegetación, la cual fue procesada en formato raster (celdas) de $100 \mathrm{~km}^{2}$, realizando así un conteo de celdas para calcular los umbrales de área definidos en este subcriterio de ADO del ecosistema. Una vez realizado esto, se aplicó el mismo subcriterio condicionante de B1, una medida de cambio en la extensión espacial definida en el criterio A2b.

\section{Criterio C - Declinación en procesos abióticos}

La degradación del componente abiótico puede tener una inferencia importante en la reducción y desaparición de ciertos nichos disponibles para especies individuales. En este sentido, la identificación de la degradación de ciertos componentes del hábitat puede permitir generar un diagnóstico del riesgo al que se encontrarían sometidos los otros componentes del sistema. Los subcriterios definidos se basan en los mismos tres periodos de tiempo del criterio A; en el caso del bosque espinoso se evaluó únicamente el subcriterio $\mathrm{C} 2$, que da cuenta de una degradación ambiental en los próximos 50 años, ya que no fue posible encontrar datos suficientes para poder evaluar los sub criterios $\mathrm{C}^{\text {y }}$ C3, por lo que la categoría asignada fue Datos Insuficientes (DD). Para aplicar el subcriterio C2, se utilizaron los resultados obtenidos en el estudio "Plan de Acción Para la Protección y Conservación de la Biodiversidad, en un Contexto de Adaptación al Cambio 
Climático" (Santibañez et al. 2013). En el estudio señalado, se calculó un índice de estrés integrado obtenido a partir de la estimación de un estrés hídrico y un estrés térmico. El concepto de estrés se refiere a una variación tanto positiva o negativa de los rangos de tolerancia bioclimática actuales respecto a un escenario futuro de cambio climático. La proyección actual se realizó utilizando ambos índices para un escenario de cambio climático al año 2050.

\section{Criterio D - Declinación en procesos e interacciones bióticas:}

El ecosistema de bosque espinoso está sujeto al sobrepastoreo. Tanto el ganado como los conejos, pueden causar un daño importante en el reclutamiento de las plantas claves que componen este ecosistema, a través de la depredación de semillas y plántulas. Aunque se conoce de esta interacción, actualmente no existen datos que estimen la extensión e intensidad de estas actividades.

\section{Evaluación final}

Se aplicaron un total de 6 subcriterios, correspondientes a los tres criterios de evaluación (A, B, C). En el caso de los criterios y subcriterios que no pudieron ser evaluados por falta de información, se les asignó la categoría de Datos Insuficientes (DD), ya que pudimos constatar que los datos no estaban disponibles. Es importante aclarar que la categoría DD es distinta a No Evaluado (NE), ya que se hace la constatación de que la información para la evaluación debe ser desarrollada.

Para el sub criterio A2b, el bosque espinoso se define como Preocupación Menor (LC), ya que se identifica una baja pérdida de superficie respecto a su área total en los últimos 20 años (4,57\%). Distinto es el caso de la pérdida histórica del bosque espinoso, la cual alcanza casi un $40 \%$ del área total del ecosistema (37,9\%), por lo que se clasifica como Vulnerable (VU) bajo el criterio A3 (Fig. 6).

En el caso del criterio B, el bosque espinoso se clasifica como Preocupación Menor (LC), tanto en el caso del subcriterio definido por el polígono mínimo convexo (al tener una superficie de $44.213 \mathrm{~km}^{2}$ ), como en el subcriterio $\mathrm{B} 2 \mathrm{a}$, al poseer una superficie remanente identificada con 60 celdas de $10 \mathrm{~km}^{2}$ (Fig. 7). El subcriterio B3 también se considera como Preocupación Menor (LC), al identificarse un mayor número de localidades que el umbral máximo definido (10 localidades).

Para el caso del Criterio C2, que se refiere a la degradación ambiental futura, se clasificó el ecosistema como Vulnerable (VU), al presentar un alto grado de estrés hídrico, combinando la extensión espacial e intensidad relativa del índice, proyectado al año 2050 (Fig. 8).

La aplicación de los criterios se resume en la Cuadro 2. Una vez aplicados todos los criterios, se define para su clasificación final aquel que presente la categoría de amenaza superior (Keith et al. 2103; Bland et al. 2016); en el caso del bosque espinoso, la categoría final identificada sería la de Vulnerable (VU), definida por los criterios A3 y C2. 
Cuadro 2. Resumen de la evaluación del Ecosistema del Espinal basado en los criterios de la lista roja de Ecosistemas de IUCN (NE, no evaluado; DD, datos deficientes; LC, sin preocupación; VU, vulnerable).

\begin{tabular}{|l|c|c|c|c|c|c|}
\hline Criterio & A & B & C & D & E & Final \\
\hline Subcriterio 1 & DD & DD & NE & NE & NE & VU \\
\hline Subcriterio 2 & LC & LC & VU & NE & & \\
\hline Subcriterio 3 & VU & NE & NE & NE & & \\
\hline
\end{tabular}

Figura 7. Distribución actual del Espinal según criterio B2a.

\section{Distribución Espinal}

Distribución Potencial

Distribucion Actual (Celdas de $10 \mathrm{~km}^{2}$ )

Poligono Minimo Convexo

\author{
(a)
}
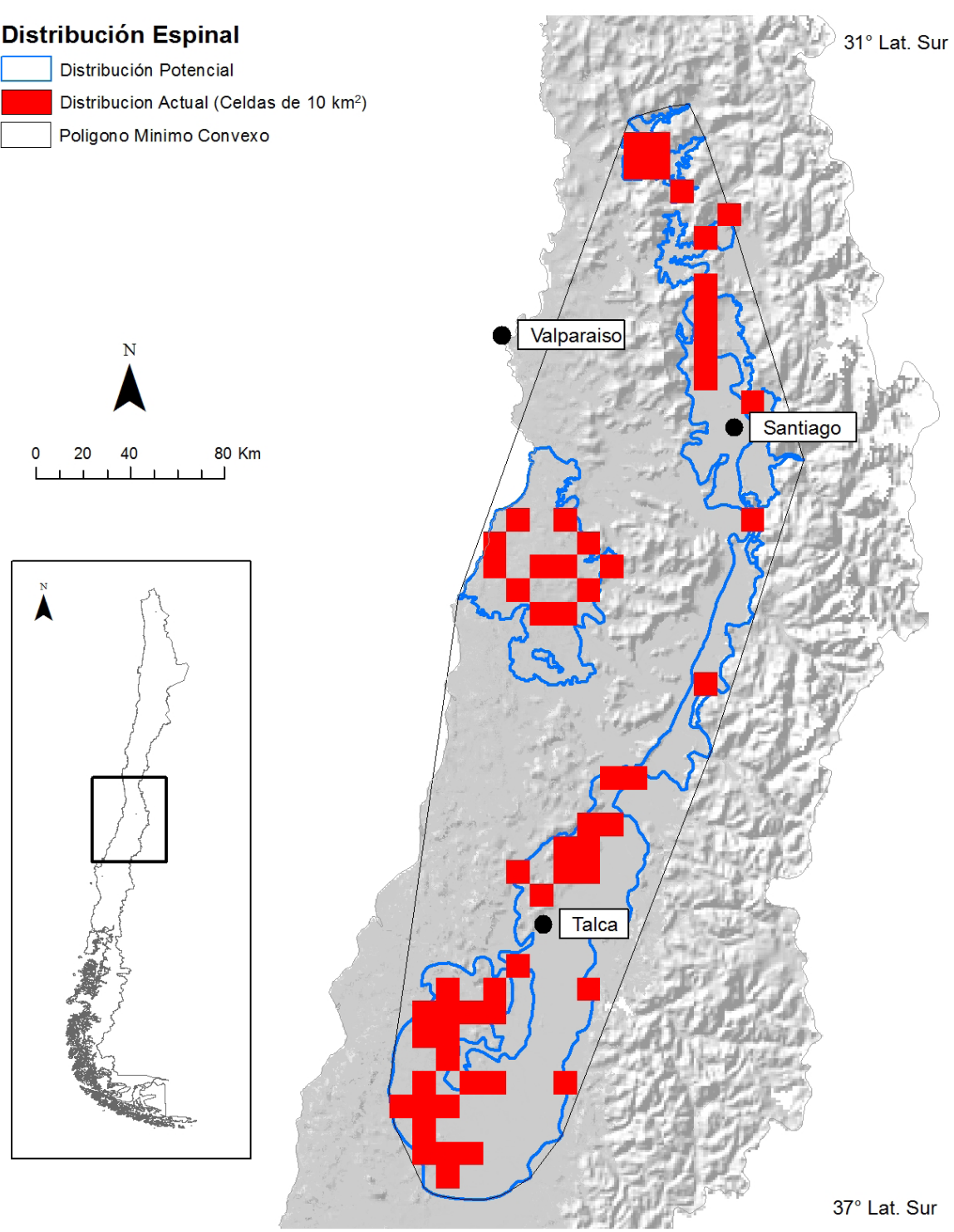

Fuente: Elaboración propia 
Figura 8. Distribución del estrés hídrico del Espinal, utilizado para la definición de criterio C2.

(Santibañez et al. 2013)

Distribución Espinal

$\square$ Distribución Potencial

Estrés Hidrico Proyección Año 2050

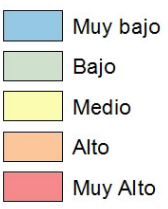

A
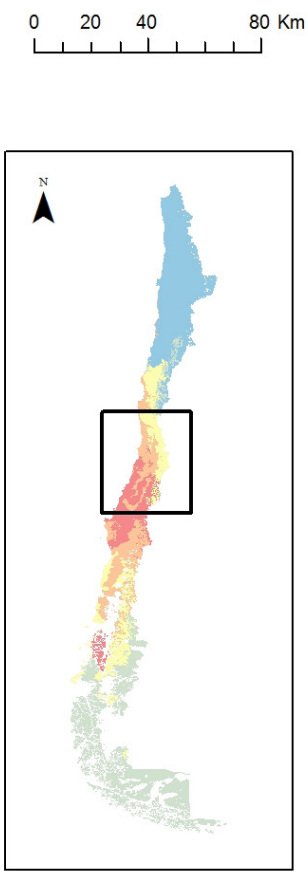

$\mathrm{Km}$
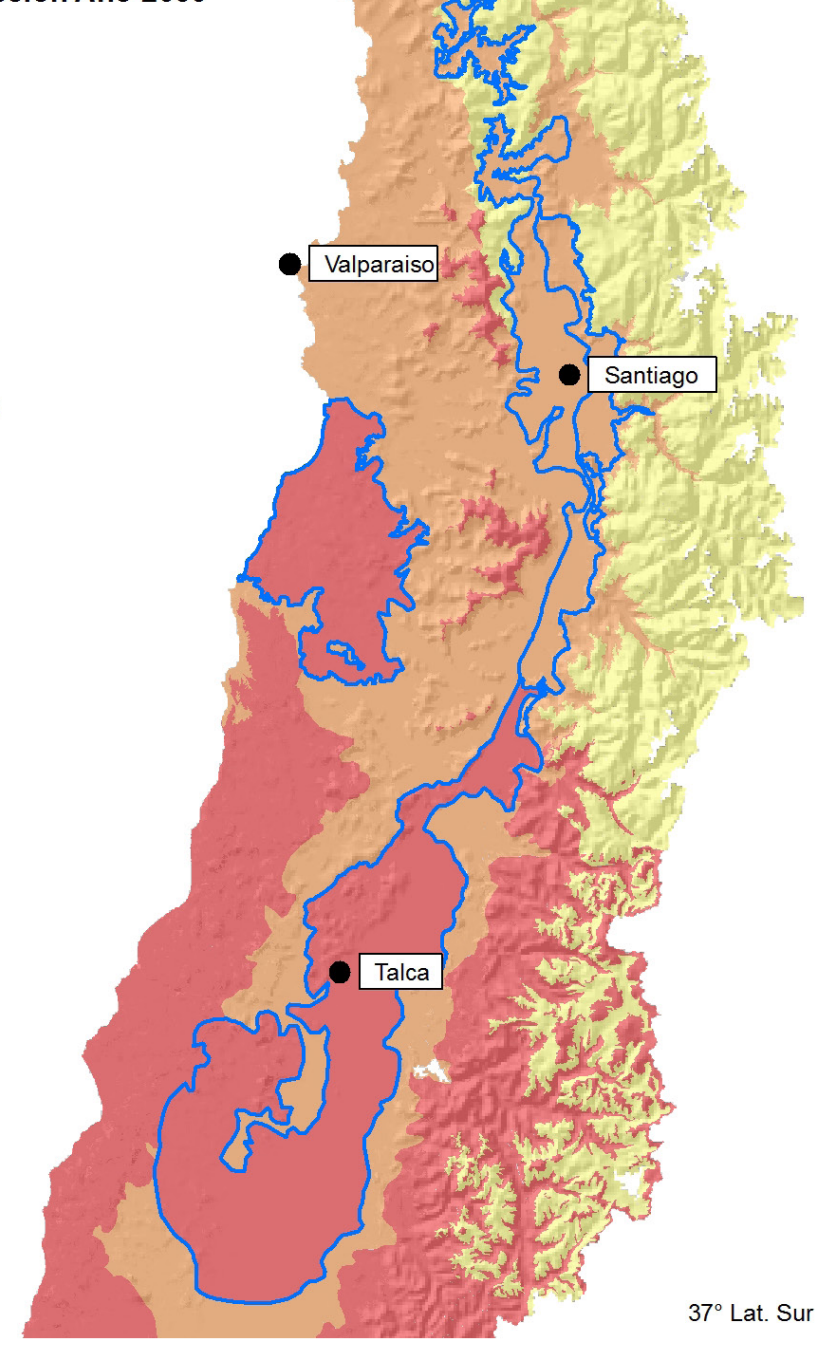

Fuente: Elaboración propia

\section{Conclusión}

Una vez realizado el ejercicio con el ecosistema de Bosque Espinoso de Chile central, se pudieron identificar tres principales limitaciones en la aplicación de la metodología de Lista Roja de Ecosistemas de UICN. La primera limitación se relaciona a la definición de los umbrales que 
definen las categorías de amenaza para cada criterio. Estos valores fueron fijados arbitrariamente en función de la opinión de expertos y de la experiencia con análisis similares realizados para especies. Es por esto que la aplicación de los criterios debería contener además de los umbrales definidos, análisis de sensibilidad de los mismos para evaluar el efecto sobre la definición de las categorías. El segundo aspecto identificado, se relaciona con la falta de claridad en la definición de las variables a utilizar para definir los criterios de funcionalidad (Criterios C y D). Aunque se reconoce que las variables asociadas a funcionalidad en los ecosistemas dependen directamente del ámbito en que este se encuentre (i.e. terrestre, marino o aguas continentales), la definición de un conjunto de variables de evaluación podría aclarar la forma de cómo abordar estos criterios y permitiría, a la vez, en avanzar hacia protocolos y metodologías que permitan levantar información del conjunto de variables definidas. Finalmente, el tercer aspecto se relaciona con los criterios asociados a distribución (A y B), donde se reconoce una relación escala-dependiente para su determinación. Es decir, la categorización final de estos criterios y algunos de sus subcriterios, podría estar relacionada con la escala espacial utilizada para definir el ecosistema.

Por otra parte, el desarrollo de este ejercicio permite identificar algunos vacíos de información que son extensibles al resto de los ecosistemas en Chile. Por ejemplo, llama la atención la escasa información levantada sobre los procesos claves de los ecosistemas, como las principales interacciones que se dan entre el componente biótico y abiótico dentro de estos (e.g., polinizadores). También se identificaron vacíos en la información en la disponibilidad de datos para los elementos abióticos que permiten evaluar el criterio C (pérdida de funcionalidad por elementos abióticos), en el caso de este ejercicio, las variables usadas en el ejemplo aplicado al criterio C2, tienen una extensión espacial nacional, la utilización de variables más locales asociadas al ecosistema, permitiría una evaluación más realista del estado actual del bosque espinoso.

Más allá de lo mencionado anteriormente, el ejercicio permitió avanzar en la caracterización y evaluación de un ecosistema que, a pesar de su relevancia cultural y cercanía con los grandes centros urbanos de Chile central, se mantiene distante tanto del interés público como de los investigadores del área ambiental. Este escaso interés se refleja en forma práctica en la mínima representación que tiene en la actualidad el Bosque Espinoso en los instrumentos oficiales de protección que posee el país, siendo identificado, por ejemplo, como la formación vegetacional con menor representación dentro del Sistema Nacional de Áreas Silvestres Protegidas del Estado (Pliscoff \& Fuentes-Castillo 2011; Moya et al. 2014; Luebert \& Pliscoff 2017).

Se espera que los antecedentes y metodologías presentadas en este ejercicio, permitan avanzar hacia el desarrollo de nuevos manejos y prácticas que favorezcan a la conservación y protección del bosque espinoso de Chile Central. Por ejemplo, existen propuestas concretas (Root-Bernstein \& Jaksic 2015) para el manejo del sistema silvopastoral del bosque espinoso y los análisis de representatividad realizados a escala nacional (Pliscoff \& Fuentes-Castillo 2011; Squeo et al. 2013; Luebert \& Pliscoff 2017), han identificado las áreas del bosque espinoso que podrían complementar al Sistema Nacional de Áreas Protegidas de Chile.

\section{Agradecimientos}

Los autores agradecen el apoyo de CONICYT (Comisión Nacional de Investigación Científica y Tecnológica) a través del proyecto PIA-Anillo Soc 1404. 


\section{Bibliografía}

AKÇAKAYA, H.R.; FERSON, S.; BURGMAN, M.A.; KEITH, D.A.; MACE, G.M. \& TODD, C.R. Making consistent IUCN classifications under uncertainty. Conservation Biology, 2000, Vol. 14, N4, p. 1001-1013.

ARROYO, M.T.K.; MARTICORENA, C.; MATTHEI, O. \& CAVIERES, L. Plant invasions in Chile: present patterns and future predictions. En: Invasive Species in a Changing World. MOONEY, H.A. Y HOBBS, R.J. (editores). Washington, D.C.: Island Press, 2000, p. 385-421.

AYENSU, E.; CLAASEN, D.V.R.; COLLINS, M.; DEARING, A.; FRESCO, L.; GADGIL, M.; GITAY, H.; GLASER, G.; JUMA, C.; KREBS, J.; LENTON, R.; LUBCHENCO, J.; MCNEELY, J.A.; MOONEY, H.A.; PINSTRUP-ANDERSEN, P.; RAMOS, M.; RAVEN, P.; REID, W.V.; SAMPER, C.; SARUKHAN, J.; SCHEI, P.; GALIZIA, J.E.; WATSON, R.T.; GUANHUA, X: \& ZAKRI, A.H. Ecology: International ecosystem assessment. Science, 1999, Vol. 286, N 5440, p. 685-686.

BARROS, H.; IZARRA, C.; MERLO, G. \& SAJO-BOHUS, L. Evaluación de la intrusión marina y salinización de acuíferos costeros en áreas piloto al noreste del estado Falcón, Venezuela. En: RIVEROS CABALLERO, M.; SÁNCHEZ, L.E. \& PAOLINI, J. (editores). Memoria Taller Binacional y Regional Sobre Desertificación. Caracas, Venezuela: Ediciones IVIC (Instituto Venezolano de Investigaciones Científicas). 2007, p. 65-70.

BLAND, L.M.; ROWLAND, J.A.; REGAN, T.J.; KEITH, D.A.; MURRAY, N.J.; LESTER, R.E.; LINN, M.; RODRÍGUEZ, J.P. \& NICHOLSON, E. Developing a standardized definition of ecosystem collapse for risk assessment. Frontiers in Ecology and Environment, 2018, Vol. 16, №1, p. 29-36.

BLAND, L.M.; KEITH, D.A.; MILLER, R.M.; MURRAY, N.J. \& RODRÍGUEZ, J.P. Directrices para la aplicación de las Categorías y Criterios de la Lista Roja de Ecosistemas de UICN, Versión 1.0. Gland, Suiza: UICN. 2016,

BURGMAN, M. Kinds of uncertainty. En: BURGMAN, M. Risks and decisions for conservation and environmental management. Cambridge: Cambridge University Press; 2005. p. 42-61.

BURGMAN, M. Values, history and perception. En: BURGMAN, M. Risks and decisions for conservation and environmental management. Cambridge: Cambridge University Press; 2005. p. 1-24.

BUTCHART, S.H.; WALPOLE, M.; COLLEN, B:; VAN STRIEN, A.; SCHARLEMANN, J.P.; ROSAMUNDE, A.E.; BAILLIE, J.E.; BOMHARD, B.; BROWN, C.; BRUNO, J.; CARPENTER, K.E.; CARR, G.M.; CHANSON, J.; CHENERY, A.M.; SIRKE, J.; DAVIDSON, N.C.; DENTENER, F.; FOSTER, M.; GALLI, A.; GALLOWAY, J.N.; GENOVESI, P.; GREGORY, R.D.; HOCKINGS, M.; KAPOS, V.; LAMARQUE, J.F.; LEVERINGTON, F.; LOH, J.; MCGEOCH, M.A.; MCRAE, L.; MINASYAN, A.; HERNÁNDEZ, M.; OLDFIELD, T.E.; PAULY, D.; QUADER, S.; REVENGA, C.; SAUER, J.R.; SKOLNIK, B.; SPEAR, D.; STANWELL-SMITH, D.; STUART, S.N.; SYMES, A.; TIERNEY, M.; TYRRELL, T.D.; VIÉ, J.C. \& WATSON, R. Global Biodiversity: Indicators of Recent Declines. Science, 2010, Vol. 328, p. 1164-1168.

CONVENTION ON BIOLOGICAL DIVERSITY (CBD). Convention on Biological Diversity, 1992, United Nations Conference on Environment and Development, Rio de Janeiro, Brazil. 
COLLEN, B.; GRIFFITHS, J.; FRIEDMANN, Y.; RODRÍGUEZ, J.P.; ROJAS-SUÁREZ, F. \& BAILLIE, J.E. Tracking Change in National-Level Conservation Status: National Red Lists. En: COLLEN B, PETTORELLI N, BAILLIE J. E. \& DURANT, S.M. (editores). Biodiversity Monitoring and Conservation: Bridging the Gap Between Global Commitment and Local Action. Hoboken, New Jersey, USA: John Wiley \& Sons, Ltd., 2013, p. 19-44.

CORNEJO, R. \& GÁNDARA, J. Influencia de la estrata arbustiva en la productividad de la estrata herbácea de la estepa de Acacia caven (Mol) Hook et Am. Memoria de título Ingeniería Forestal. Universidad de Chile. Facultad de Ciencias Agrarias y Forestales, 1980, Santiago, Chile.

COWLING, R.M.; KNIGHT, A.T.; FAITH, D.P.; FERRIER, S.; LOMBARD, A.T.; DRIVER, A.; ROUGET, M.; MAZE, K. \& DESMET, P.G. Nature conservation requires more than a passion for species. Conservation Biology, 2004, Vol. 18, Nº 6, p. 1674-1676.

CURRAN, L.M. \& TRIGG, S.N. Sustainability science from space: Quantifying forest disturbance and land-use dynamics in the Amazon. Proceedings of the National Academy of Sciences of the United States of America, 2006, Vol. 103, №34, p. 12663-12664.

DIRZO, R. \& RAVEN, P.H. Global state of biodiversity and loss. Annual Review of Environment and Resources, 2003, Vol. 28, p. 137-167.

FERRIER, S. Mapping spatial pattern in biodiversity for regional conservation planning: Where to from here? Systematic Biology, 2002, Vol. 51, N² 2, p. 331-363.

FOLKE, C.; CARPENTER, S.; WALKER, B.; SCHEFFER, M.; ELMQVIST, T.; GUNDERSON, L. \& HOLLING, C.S. Regime shifts, resilience, and biodiversity in ecosystem management. Annual Review of Ecology Evolution and Systematics, 2004, Vol. 35, p. 557-581.

FUENTES, E.R.; AVILÉS, R. \& SEGURA, A. Landscape change under indirect effects of human use: The Savanna of Central Chile. Landscape Ecology, 1989, Vol. 2, p. 73-80.

FUNK, S.M. \& FA, J.E. Ecoregion prioritization suggests an armoury not a silver bullet for conservation planning. PLoS One, 2010, Vol. 5, № 1, p. e8923.

GAJARDO, R. La vegetación natural de Chile. Clasificación y Distribución Geográfica. Editorial Universitaria, Santiago, 1994.

GASTON, K.J. \& FULLER, R.A. Commonness, population depletion and conservation biology. Trends in Ecology \& Evolution, 2008, Vol. 23, № 1, p. 14-19.

GUTIÉRREZ, J.R.; HOLMGREN, M.; MANRIQUE, M. \& SQUEO, F.A. Reduced herbivore pressure under rainy ENSO conditions could facilitate dryland reforestation. Journal of Arid Environments, 2007, Vol. 68, p. 322-330.

HOBBS, R.J.; ARICO, S.; ARONSON, J.; BARON, J.S.; BRIDGEWATER, P.; CRAMER, V.A.; EPSTEIN, P.R.; EWEL, J.J.; KLINK, C.A.; LUGO, A.E.; NORTON, D.; OJIMA, D.; RICHARDSON, D.M.; SANDERSON, 
E.W.; VALLADARES, F.; VILA, M.; ZAMORA, R. \& ZOBEL, M. Novel ecosystems: theoretical and management aspects of the new ecological world order. Global Ecology and Biogeography, 2006, Vol. 15, No 1, p. 1-7.

HOEKSTRA, J.M.; BOUCHER, T.M.; RICKETTS, T.H. \& ROBERTS, C. Confronting a biome crisis: global disparities of habitat loss and protection. Ecology Letters, 2005, Vol. 8, № 1, p. 23-29.

HOOPER, D.U.; CHAPIN, F.S.; EWEL, J.J.; HECTOR, A.; INCHAUSTI, P. LAVOREL, S.; LAWTON, J.H.; LODGE, D.M.; LOREAU, M.; NAEEM, S.; SCHMID, B.; SETALA, H.; SYMSTAD, A.J.; VANDERMEER, J. \& WARDLE, D.A. Effects of biodiversity on ecosystem functioning: A consensus of current knowledge. Ecological Monographs, 2005, Vol. 75, N 1, p. 3-35.

JAX, K. Function and "functioning" in ecology: what does it mean? Oikos, 2005, Vol. 111, № 3, p. 641-648.

KEITH, D. A.; RODRÍGUEZ, J. P.; BROOKS, T. M.; BURGMAN, M. A.; BARROW, E. G.; BLAND, L.; COMER, P.; FRANKLIN, J.; LINK, J.; MCCARTHY, M.; MILLER, R.; MURRAY, N.; NEL, J.; NICHOLSON, E.; OLIVIERA-MIRANDA, T.; REGAN, T.; RODRIGUEZ-CLARK, K., ROUGET, M.\& SPALDING, M. D. The IUCN Red List of Ecosystems: Motivations, Challenges, and Applications. Conservation Letters, 2015, Vol.8, N³, p. 214-226.

KEITH, D.A.; RODRÍGUEZ, J.P.; RODRÍGUEZ-CLARK, K.M.; NICHOLSON, E.; AAPALA, K.; ALONSO, A.; ASMUSSEN, M.; BACHMAN, S.; BASSET, A.; BARROW, E.G.; BENSON, J.S.; BISHOP, M.J.; BONIFACIO, R.; BROOKS, T.M.; BURGMAN, M.A.; COMER, P.; COMÍN, F.A.; ESSL, F.; FABER-LANGENDOEN, D.; FAIRWEATHER, P.G.; HOLDAWAY, R.J.; JENNINGS, M.; KINGSFORD, R.T.; LESTER, R.E.; NALLY, R.M.; MCCARTHY, M.A.; MOAT, J.; OLIVEIRA-MIRANDA, M.A.; PISANU, P.; POULIN, B.; REGAN, T.J.; RIECKEN, U.; SPALDING, M.D. \& ZAMBRANO-MARTÍNEZ, S. 2013. Scientific Foundations for an IUCN Red List of Ecosystems. PLoS One 8(5): e62111.

LOREAU, M. Linking biodiversity and ecosystems: towards a unifying ecological theory. Philosophical Transactions of the Royal Society B-Biological Sciences, 2010, Vol. 365, No 1537, p. 49-60. LUEBERT, F. \& PLISCOFF, P. Sinopsis bioclimática y vegetacional de Chile, Segunda Edición. Editorial Universitaria, 2017.

MACE, G.M.; COLLAR, N.J.; GASTON, K.J.; HILTON-TAYLOR, C.; AKCAKAYA, H.R.; LEADER-WILLIAMS, N.; MILNER-GULLAND, E.J. \& STUART, S.N. Quantification of Extinction Risk: IUCN's System for Classifying Threatened Species. Conservation Biology, 2008, Vol. 22, Nº 6, p. 1424-1442.

MOYA, D.; HERREROS, J. \& FERREYRA, J. Representatividad actual de los pisos vegetacionales en el Sistema Nacional de Áreas Protegidas y de sitios prioritarios para la conservación en Chile. Documento de Trabajo. Proyecto MMA / GEF-PNUD Creación de un Sistema Nacional de Áreas Protegidas para Chile: Estructura Financiera y Operacional. Santiago, Chile, 2014.

MUÑOZ-PEDREROS, A.; FLETCHER, S.; YÁÑEZ, J. \& SÁNCHEZ, P. Diversidad de micromamíferos en tres ambientes de la Reserva Diversity of small mammals in three environments of the National Reserve Lago. Gayana, 2010, Vol. 74, p. 1-11. 
MYERS, N.; MITTERMEIER, R.A.; MITTERMEIER, C.G.; DA FONSECA, G.A. \& KENT, J. Biodiversity hotspots for conservation priorities. Nature, 2000, Vol. 403, p. 853-858.

NICHOLSON, E.; KEITH, D.A. \& WILCOVE, D.S. Assessing the threat status of ecological communities. Conservation Biology, 2009, Vol. 23, Nº 3, p. 259-274.

NIJMAN, V. Decline of the endemic Hose's langur Presbytis hosei in Kayan Mentarang National Park, east Borneo. Oryx, 2005, Vol. 39, № 2, p. 223-226.

OBERDORFER, E. Die Wiesenlandschaft Süd-Chiles. Umschau in Wiessenschaft und Technick (N¹2), 1960. p. $370-372$

OLSON, D.M. \& DINERSTEIN, E. The Global 200: Priority ecoregions for global conservation. Annals of the Missouri Botanical Garden, 2002, Vol. 89, № 2, p. 199-224.

OVALLE, C. \& GODRON, M. Influencia del árbol sobre la vegetación herbácea en matorrales de "Acacia caven". Rol del animal (ovinos) en las interacciones árbol-pasto. Studia Oecológica, 1989, Vol. 6, p. 225-244.

PATRICK, W.S.; SPENCER, P.; LINK, J.; COPE, J.; FIELD, J.; KOBAYASHI, D.; LAWSON, P.; GEDAMKE, T.; CORTES, E.; ORMSETH, O.; BIGELOW, K. \& OVERHOLTZ W. Using productivity and susceptibility indices to assess the vulnerability of United States fish stocks to overfishing. Fishery Bulletin, 2010, Vol. 108, Nº 3, p. 305-322.

PAVEZ, E.F.; LOBOS, G.A. \& JAKSIC, F.M. Long-term changes in landscape and in small mammal and raptor assemblages in central Chile. Revista Chilena de Historia Natural, 2010, Vol. 83, p. 99111.

PICKETT, S.T. \& CADENASSO, M.L. The ecosystem as a multidimensional concept: Meaning, model, and metaphor. Ecosystems, 2002, Vol. 5, No 1, p. 1-10.

PLISCOFF, P. Aplicación de los criterios de la Unión Internacional para la Conservación de la Naturaleza (IUCN) para la evaluación de riesgo de los ecosistemas terrestres de Chile. Ministerio del Medio Ambiente. Santiago, Chile, 2015.

PLISCOFF, P., \& FUENTES-CASTILLO, T. Representativeness of terrestrial ecosystems in Chile's protected area system. Environmental Conservation, 2011, Vol. 38, №3, p. 303-311.

RODRIGUEZ, J.P.; KEITH, D.A.; RODRIGUEZ-CLARK, K.M.; MURRAY, N.J.; NICHOLSON, E.; REGAN, T.J.; MILLER, R.M.; BARROW, E.G.; BLAND, L.M.; BOE, K.; BROOKS, T.M.; OLIVEIRA-MIRANDA, M.A.; SPALDING, M. \& WIT, P. A practical guide to the application of the IUCN Red List of Ecosystems criteria. Philosophical Transactions of the Royal Society B-Biological Sciences, 2015, Vol. 370, p. 20140003

RODRIGUEZ, J.P.; RODRIGUEZ-CLARK, K.M.; BAILLIE, J.E.; ASH, N.; BENSON, J.; BOUCHER, T.; BROWN, C.; BURGESS, N.D.; COLLEN, B.E.; JENNINGS, M.; KEITH, D.A.; NICHOLSON, E.; REVENGA, C.; REYERS, B.; ROUGET, M.; SMITH, T.; SPALDING, M.; TABER, A.; WALPOLE, M.; ZAGER, I. \& ZAMIN, 
T. Establishing IUCN red list criteria for threatened ecosystems. Conservation Biology, 2011, Vol. $25, N^{\circ} 1$, p. 21-29.

ROOT-BERNSTEIN, M. \& SVENNING, J. Restoring connectivity between fragmented woodlands in Chile with a reintroduced mobile link species. Perspective in Ecology and Conservation, 2017, Vol. 15, 292-299.

ROOT-BERNSTEIN, M., \& JAKSIC, F. M. Ecosystem process interactions between central Chilean habitats. Global Ecology and Conservation, 2015, Vol. 3, p. 776-788.

ROOT-BERNSTEIN, M., \& JAKSIC, F. The Chilean Espinal: Restoration for a Sustainable Silvopastoral System. Restoration Ecology, 2013, Vol. 21, N4, p. 409-414.

SANTIBAÑEZ, F.; SANTIBAÑEZ, P.; CAROCA, C.; GONZÁLEZ, P.; GAJARDO, N.; PERRY, P.; SIMONETTI, J. \& PLISCOFF, P. Plan de acción para la protección y conservación de la biodiversidad, en un contexto de adaptación al cambio climático, Ministerio de Medio Ambiente, Chile, 2013.

SERRA, M. Chile: Acacia caven. En: FAO: Especies arbóreas y arbustivas para las zonas áridas y semiáridas de América Latina, 1997, p. 159-167.

SQUELLA, F. \& SOTO, G. Desarrollo de sistemas agroforestales en la zona de tendencia desértica y mediterránea árida y semiárida de Chile. Serie Documentos Técnicos N 5, 1993.

SQUEO, F.; ESTÉVEZ, R. A.; STOLL, A.; GAYMER, C. F.; LETELIER, L. \& SIERRALTA, L. Towards the creation of an integrated system of protected areas in Chile: achievements and challenges. Plant Ecology \& Diversity, 2012, Vol. 5, N² 2, p. 233-243.

SRIVASTAVA, D.S. \& VELLEND, M. Biodiversity-ecosystem function research: Is it relevant to conservation? Annual Review of Ecology Evolution and Systematics, 2005, Vol. 36: 267-294.

STODDARD, J.L.; HERLIHY, A.T.; PECK, D.V.; HUGHES, R.M.; WHITTIER, T.R. \& TARQUINIO, E. A process for creating multimetric indices for large-scale aquatic surveys. Journal of the North American Benthological Society, 2008, Vol. 27, N 4, p. 878-891.

TANSLEY, A.G. The use and abuse of vegetational concepts and terms. Ecology, 1935, Vol. 16, p. 284-307.

TIERNEY, G.L.; FABER-LANGENDOEN, D.; MITCHELL, B.R.; SHRIVER, W.G. \& GIBBS, J.P. Monitoring and evaluating the ecological integrity of forest ecosystems. Frontiers in Ecology and the Environment, 2009. Vol. 7, № 6, p. 308-316.

TILMAN, D., CLARK, M., WILLIAMS, D.R., KIMMEL, K., POLASKY, S., PACKER, C. Future threats to biodiversity and pathways to their prevention. Nature, 2017, 546, p.73-81.

UICN. Categorías y Criterios de la Lista Roja de la UICN: Versión 3.1. Comisión de Supervivencia de Especies de la Unión Mundial para la Naturaleza (UICN), Gland, Suiza y Cambridge, Reino Unido. 2001 
UICN. The IUCN Red List of Threatened Species. Obtenido junio 11, 2018, desde http://www.iucn. org/about/work/programmes/species/our_work/the_iucn_red_list/.

VALDIVIA, C.E. \& ROMERO, C.R. En La Senda De La Extinción: El Caso Del Algarrobo Prosopis Chilensis (Fabaceae) y El Bosque Espinoso En La Región Metropolitana De Chile Central. Gayana Botanica, 2013, Vol. 70, p. 57-65.

VIÉ, J.C.; HILTON-TAYLOR, C. \& STUART, S.N. Wildlife in changing world: An analysis of the 2008 IUCN Red List of Threatened Species. IUCN, Gland, Switzerland, 2009.

WILLIS, A.J. Forum. Functional Ecology, 1997, Vol. 11, № 2, p. 268-271.

WILSON, K.A.; CARWARDINE, J. \& POSSINGHAM, H.P. Setting Conservation Priorities. Annals of the New York Academy of Sciences, 2009, Vol. 1162, p. 237-264.

ZAMIN, T.; BAILLIE, J.E.; MILLER, R.; RODRIGUEZ, J.P.; ARDID, A. \& COLLEN, B. National red listing beyond the 2010 target. Conservation Biology, 2010, Vol. 24, № 4, p. 1012-1020. 